\title{
Editorial
}

\section{Acknowledgement to Reviewers of Molecules in 2014}

Molecules Editorial Office, MDPI AG, Klybeckstrasse 64, CH-4057 Basel, Switzerland

Published: 7 January 2015

The editors of Molecules would like to express their sincere gratitude to the following reviewers for assessing manuscripts in 2014:

\begin{tabular}{|c|c|c|}
\hline Abashev, G. G. & Ågren, $\mathrm{H}$. & Alcântara, Ana C. S. \\
\hline Abass, Khaled & Agrios, Alexander G. & Alcaraz, Gilles \\
\hline Abbate, Sergio & Agrofoglio, Luigi A & Aldabbagh, Fawaz \\
\hline Abbehausen, Camilla & Aguilar, Cristóbal N. & Aldrich-Wright, Janice R \\
\hline Abdelmohsen, Usama & Aguirre, Pedro & Alexopoulos, A. \\
\hline Ramadan & Ahn, Joong-Hoon & Alezra, Valérie \\
\hline Abdullayev, Elshad & Ahn, Kyo Han & Algar, W. Russ \\
\hline Abe, Koichi & Ahn, Soon-Cheol & Ali, Shaikh A. \\
\hline Abe, Naohito & Ahrends, Robert & Ali, Simi \\
\hline Abe, Ryu & Aidoudi, Farida H. & Alisi, Anna \\
\hline Abel, S. & Ainslie, Kristy M. & Allcock, Harry R. \\
\hline Åberg, Ola & Aires, Alfredo & Allemann, Rudolf K. \\
\hline Abliz, Zeper & Ajikumar, Parayil Kumaran & Allen, N.S. \\
\hline Acero, Nuria & Akagawa, Mitsugu & Allen, William J. \\
\hline Achelle, Sylvain & Akai, Shuji & Allgaier, Jürgen \\
\hline Adam, Rosa & Akira, Kazuki & Almajano, María Pilar \\
\hline Adam, Vojtech & Alabugin, Igor V. & Al-Masum, Mohammad \\
\hline Adams, Joshua & Alam, Mohammad A. & Almeida, Wanda \\
\hline Adams, Michael & Alam, M S. & Almerico, Anna Maria \\
\hline Adams, Timothy E. & Alami, Jones & Almqvist, Fredrik \\
\hline Adeloye, Adewale O. & Alavez, Silvestre & Al-Musayeib, Nawal M. \\
\hline Adriano, Marocco & ALBERICIO, Fernando & Al-Obaidi, Hisham \\
\hline Afarinkia, Kamyar & Albert, Nick & Alonsoa, Francisco \\
\hline Afiyatullov, Shamil Sh. & Albini, Angelo & Al-Othman, Zeid \\
\hline Afonso, Carlos A.M. & Albrectsen, Benedicte R. & Altman, Ryan A. \\
\hline Agamennone, Mariangela & Albuquerque, Magaly & Alvarado, Vladimir \\
\hline
\end{tabular}


Alvarez-Builla, Julio

Alvarez-Lorenzo, Carmen

Alvarez-Suarez, José Miguel

Alves, Gilberto

Alves, Isabel D.

Alves, Maria José

Amadelli, R.

Amarasekara, Ananda S.

Ambudkar, Suresh V.

Amić, Dragan

Amo-Ochoa, Pilar

Ananikov, Valentine P.

Anastyuk, Stanislav D.

Anderson, Marilyn

Andjelkovic, Anuska V.

Andrade, Carolina Horta

Andrade, Leandro

Andrade, Paula Andrade

Andrade, Suzana M.

Andrade-Cetto, Adolfo

Andreoli, Roberta

Andres, Juan

Andrushchenko, Valery

Andújar, Isabel

Angela, Bisio

Angulo, Javier

Annaert, Pieter

Anraku, Makoto

Antelmann, Haike

Anthony, Okoh I

Antonchick, Andrey

Anwar, Sri Haryani

Aoyagi, Noboru

Apak, Reşat

Apte, Suneel S.

Arai, Masayoshi

Arai, Takayoshi

Arapitsas, Panagiotis

Araújo, Marcelo Gonzaga

de Freitas

Araujo, Maria T. de

Arbault, Stephane
Arbona, Vicent

Arce-Estrada, Elsa M.

Arceusz, Agnieszka

Archibald, Steve J.

Arena, C.

Arias-Pérez, María-Selma

Ariga, Hiroyoshi

Ariga, Katsuhiko

Arion, Vladimir

Ariyama, Kaoru

Armentano, Donatella

Armishaw, Christopher J.

Arnason, John T.

Arnson, Yoav

Arrabal, Carlos

Arranz, Elena

Arrieta, Jesús

Arsu, Nergis

Artese, Anna

Aruoma, Okezie

Asai, Teigo

Asakawa, Yoshinori

Asano, Keisuke

Ashton, Kevin

Assadi, Aymen Amine

Assfeld, Xavier

Ata, Athar

Atanasov, Atanas G.

Athayde, Margareth Linde

Atkinson, Ross G.

Attoub, Samir

Aubé, Jeffrey

Augugliaro, Vincenzo

Avarvari, Narcis

Awale, Suresh

Awual, M. Rabiul

Azémaa, Joëlle

Azuma, Akifumi

Bach, Horacio

Bacher, Adelbert

Bácskay, Ildikó

Baczek, Tomasz
Badawi, Michael

Badinga, Lokenga

Baek, Nam In

Bagla, Victor

Bai, Gang

Bai, Xiaochun

Bailey, David

Bailly, Christian

Bakare, Oladapo

Balaz, Milan

Bald, Ilko

Balemba, Onesmo B.

Ball, Zachary T.

Baltina, L. A.

Balzarini, Jan

Bana, Emilie

Banerjee, Sanjeev

Banfi, Luca

Bangoura, Berit

Banister, Samuel

Bankova, Vassya

Bannwarth, Markus B.

Bao, Jin-Ku

Barakate, Abdellah

Baraldi, Pier Giovanni

Baranac-Stojanovi, Marija

Barbayianni, Efrosini

Barbera, Mariagnese

Barbosa, Luiz C.A.

Barceló, Francisca

Barea, Carlos

Barker, David

Barkley, Noelle

Barolo, Claudia

Baron, V T

Barrajón-Catalán, Enrique

Barreira, João

Barreiro, Eliezer Jesús

Barrena, Esther

Barreto, Maria Do Carmo

Barrett, Ann

Barrio, Pablo 
Barros, Lillian

Barth, Brian M.

Basak, Saroj K.

Basha, Riyaz

Basini, Giuseppina

Bassyouni, Fatma A.

Batalla, Pilar

Batchelor, Warren

Bates, Philip D.

Battino, Maurizio

Baxendale, Ian

Bayindir, Zerrin Sezgin

Baykal, A.

Baziard, Geneviève

Bazylak, Grzegorz

Bazylińska, Urszula

Bazylko, Agnieszka

Bazzocchi, Isabel L.

Beauregard, Marc

Beck, Stephanie

Beckmann, Manfred

Bednarczyk-Cwynar, Barbara

Bednarski, Patrick

Beeren, Sophie

Beerhues, Ludger

Begum, Shamim A.

Bekhit, Aladin

Beletskaya, Irina P.

Belfield, Kevin

Belfort, Georges

Bell, Stephen

Bello, Nicholas T.

Belov, Georgiy

Bełtowski, Jerzy

Ben-Arie, Ruth

Benavides, Jorge

Benbrook, Doris Mangiaracina

Benelli, Giovanni

Benes, Petr

Benhida, Rachid

Béni, Szabolcs

Benini, Stefano
Benoist, Eric

Benoist, Hervé

Beránek, Radim

Berenguer-Murcia, Ángel

Bergheim, Ina

Beringhs, André O'Reilly

Bernardo-Gil, M. Gabriela

Bernini, Roberta

Bernot, Randall

Berruga, Maria Isabel

Berry, John

Berthod, Alain

Bertini, Luca

Berton, Paula

Besada, Cristina

Bescond, J.

Besson, Thierry

Bester, Megan J.

Beutler, John A.

Bhadani, Avinash

Bhagavathy, Ganga

Bhowmik, Pradip

Biagini, Giuseppe

BIAN, Zhaoxiang

Bianchi, Antonio

Biasutto, Lucia

Bielawski, Christopher W.

Biesaga, Magdalena

Bijak, Michał

Billard, Thierry

Bin, $\mathrm{Fu}$

Biot, Christophe

Birch, Edward

Birkedal, V.

Birney, David M.

Bishop-Hurley, Sharon

Bizukojc, Marcin

Bizzarri, Anna Rita

Blade Segara, Maria Cinta

Blanchard, Scott C.

Blanco, Rosa M

Blandino, $\mathrm{G}$
Blank, Kerstin

Bledzki, Andrzej K.

Bocchinfuso, Gianfranco

Boechat, Nubia

Boess, Frank G.

Bofill, Josep Maria

Bogár, Krisztián

Bogel-Łukasik, Rafal

Böhm, Volker

Boido, Vito

Boligon, Aline Augusti

Bolm, Carsten

Bonaccorsi, Ivana

Bondar, Mykhailo V.

Bonnet, Sylvestre

Bontemps, Nataly

Booth, Stephanie A.

Boparai, Hardiljeet

Boratynski, Janusz

Boratyński, Przemysław

Borges, Anabela

Borges, João P.M.R.

Boros, Eszter

Borowska, Marta

Borrego, Eli

Bouajila, Jalloul

Boué, Steve

Bouquillon, Sandrine

Bourgault, Steve

Bourgeois, Dominique

Bou-Saab, Hamid

Bousbaa, Hassan

Boylan, Fabio

Bradshaw, Tracey D.

Braeuer, Andreas

Braga, Valdir A.

Braissant, O.

Brancale, Andrea

Brandão, Maria das Graças

Bratkovic, Tomaz

Bratsos, Ioannis

Braun, Ruedi K. 
Bravo, Laura

Bravo-Díaz, Carlos

Brazzelli., V

Brecker, Lothar

Breeman, Wouter A.P.

Breinbauer, Rolf

Breitenfeld Granadeiro, Luiza

Bremner, John B.

Brenna, Elisabetta

Brenndörfer, Erwin

Brigante, Marcello

Brobeil, Alexander

Broberg, Anders

Brock, Matthias

Broderick, Patricia A.

Bromann, Kirsi

Bromke, Mariusz A.

Bronze, Maria

Brooke, Basil

Brown, Alan B.

Brown, Alex

Brown, J. E.

Brown, Lindsay

Browne, Wesley R.

Bruce, Duncan

Bruckner, Christian

Brudzynski, Katrina

Brun, Reto

Brunetti, Cecilia

Brüning, Ansgar

Bruno, Maurizio

Brycki, Bogumił

$\mathrm{Bu}$, Xianzhang

Buffon, Andréia

Bukowska, Bożena

Bulgariu, Laura

Buolamwini, John K.

Burketova, L

Burkholz, Torsten

Buron, Frédéric

Burritt, David

Burt, Sara A.
Buszewski, Bogus $\AA$,aw

Bystrowska, Beata

Cabaleiro, Noelia

Caboni, Pierluigi

Cabrera, Margarita Gutiérrez

Cadierno, Victorio

Cahill, Paul

Cai, Jianfeng

Cai, Kaiyong

Cai, Wenqing

Cai, Xiang-hai

Cai, Yang

Calani, Luca

Calderon, Angela

Calderone, Richard

Calderone, Vincenzo

Calero, Nuria

Calhelha, Ricardo C.

Caliandro, Rocco

Caliceti, Paolo

Caliendo, Giuseppe

Calin, George A.

Calín-Sánchez, Ángel

Calla-Magariños, J.

Callery, Patrick S.

Calligaris, Sonia

Calogeropoulou, Theodora

Calokerinos, Anthony

Camacho, M. Encarnación

Camara, Bilal

Câmara, José

Camerel, Franck

Cameron, Luiz-claudio

Campagna, Francesco

Campagne, Jean-Marc

Campiglia, Pietro

Campillo, Nuria E.

Campos, Patrícia M. B. G. Maia

Canas, Sara

Cantí, Carles

Cao, Shugeng

Cao, Wei
Cao, Xueli

Capasso, Andrea

Carbone, Anna

Carbone, $\mathrm{K}$.

Carbonell, Pablo

Carbonell-Bejerano, Pablo

Cardeno, Ana

Cardona, Wilson

Cardoso, Susana M.

Carell, Thomas

Caretto, Sofia

Carloni, Paolo

Carlsson, Uno

Carluccio, Maria Annunziata

Carnero, Amancio

Caro, Andres A.

Carpene, Christian

Carradori, Simone

Carraher Jr., Charles E.

Carrasco, Cristina

Carrier, Danielle Julie

Carro-Díaz, A.m.

Carrubba, Alessandra

Carta, Antonio

Carta, Fabrizio

Carvalho, Luiz Joaquim

Castelo Branco

Casas, Maria Isabel

Casassa, Luis Federico

Cascio, Michael

Casella, Luigi

Caselli, Alessandro

Castaneda, Homero

Castellari, Massimo

Castilho, Marcelo

Castrén, Maija L.

Castro, Angeles

Castro, Eduardo

Catalá, Myriam

Caulton, Kenneth

Cavada, Benildo

Cavalcanti, Bruno 
Cavalli, Roberta

Cavar, Sanja

Cebolla, Vicente L.

Celia, Christian

Cellini, Francesco

Cenac, Nicolas

Cenci, Elio

Cerecetto, Hugo

Cermak, R.

Černík, Miroslav

Cerra, M.c.

Ceruso, Mariangela

Cesare, Angela Di

Céspedes, Carlos

Cha, Hyukjin

Chachua, Tamar

Chae, Han-Jung

Chai, Han-Ha

Chai, Yifeng

Chaimbault, Patrick

Champagne, Benoît

Chamulitrat, Walee

Chan, Shun-Wan

Chan, Thomas

Chandra, Dhyan

Chang, Chang-Tang

Chang, Cicero L. T.

Chang, Fang-Rong

Chang, Gwo-Jyh

Chang, Hsieh

Chang, Hsueh-Wei

Chang, Il-moo

Chang, Long-Sen

Chang, Rakwoo

Chang, Shang-Tzen

Chang, Wei-chiao

Chang, Woo-Suk

Chang, Yuan-Shiun

Chang, Yung-Ho

Chaniotakis, Nikos

Chatre, Laurent

Chaves, Mariana H.
Chaves, Susana Rodrigues

Chavez, Ferman

Che, Chun-Tao

Chemat, Farid

Chen, Banglin

Chen, Bo

Chen, Chao

Chen, Chi

Chen, Chiung-Mei

Chen, Chung-Yi

Chen, Feng

Chen, Fenghua

Chen, Fusheng

Chen, Haifeng

Chen, Hao

Chen, Hong-Li

Chen, Hubiao

Chen, Huixiong

Chen, Jih-Jung

Chen, Jyh-Ping

Chen, Kew-Yu

Chen, Kuan-chou

Chen, Lanming

Chen, Ligong

Chen, Lih-Geeng

Chen, Pai-Shan

Chen, Pin-Shern

Chen, Qiao-Hong

Chen, Qi-He

Chen, Qing-Xi

Chen, Shao-Yu

Chen, Shih-Yuan

Chen, Wansheng

Chen, Wei-Jen

Chen, Wen Yong

Chen, Wen-bin

Chen, Xi

Chen, Xiao-Qing

Chen, Xiaoyan

Chen, Xin

Chen, Xiuping

Chen, Yen-Chou
Chen, Ying

Chen, Yongli

Chen, $\mathrm{Yu}$

Chen, Yunfeng

Chen, Zhonghua

Cheng Lian Ee, Gwendoline

Cheng, Heung-Chin

Cheng, Juei-tang

Cheng, Kuan-chen

Cheng, Po-Ching

Cheng, Ran

Cheng, Tak

Cheng, Xinlai

Cheng, Yongxian

Cheng, Zhi-Hui

Cherng, Jong-yuh

Cherniack, E. Paul

Cheung, Randy Chi Fai

Chevalot, Isabelle

Chew, Eng-Hui

Chi, Ki-Whan

Chi, Robin Yonggui

Chia, Win-Long

Chiacchio, Ugo

Chianese, Giuseppina

Chiang, Po-Chang

Chiang, Shu-Hua

Chiantore, Oscar

Chiashi, Shohei

Chieli, Elisabetta

Chien, Shih-Chang

Chilin, Adriana

Chiocchia, Gilles

Chiolerio, Alessandro

Chiou, Hui-ling

Chiou, Shih-hwa

Chiriano, Gianpaolo

Chirumbolo, Salvatore

Chiu, Chien-Chih

Chiu, Tsai-Hsin

Cho, Chang-Won

Cho, Hoon 
Cho, Man-Ho

Cho, Sung Yun

Cho, Sunghee

Cho, William

Chobot, Vladimir

Choi, Chang-Hwan

Choi, Hyeok

Choi, Hyoung Jin

Choi, Hyung-kyoon

Choi, Je-Min

Choi, Sanghoon

Choi, Yongseok

Choi, Young Hae

Choi, Yung-Hyun

Cholewinski, Grzegorz

Chong, Youhoon

Chorianopoulos, Nikos G.

Chou, Chang-hung

Chou, Tsui-Fen

Chow, Moses Sing Sum

Chrissanthopoulos,

Athanassios

Christov, Christo Z.

Chrobok, A.

Chrzumnicka, E.

Chu, Fang-hua

Chu, Ivan K.

Chu, Qianli

Chuang, John Shih-ching

Chuang, Ta-hsien

Chuburu, Françoise

Chui, Wai Keung

Chun, Wanjoo

Chung, Hoi Sung

Chyau, Charng-Cherng

Ciappellano, Salvatore

Cicero, Arrigo F.G.

Cichero, Elena

Cidade, Honorina

Cieśla, Łukasz

Církva, Vladimír

Citterio, Barbara
Ciuffi, Kátia J.

Clark, Lindsay V.

Claudia, Grothe, $P$

Clericuzio, Marco

Coasne, Benoit

Coates, Christopher J

Cobb, Alexander

Cock, Ian

Coelho, Elisabete

Coelho, Jorge F.

Coelho, José A

Coelho, Paulo

Coiffard, Laurence J M

Coimbra, Eliane Fields

Colacino, Joseph M.

Coles, Gerald

Coley, Helen M.

Coll, Josep

Collier, C Patrick

Collina, Simona

Collins, Maurice

Colombo, Maria Laura

Colorado, Jhonny

Colotti, Gianni

Coman, Gigi

Combet, Emilie

Concannon, Caoimhin

Conde, José

Condorelli, Momina

Conti, Barbara

Cooper, Arthur

Corbi, Pedro P.

Cordoba-Diaz, D.

Corke, Harold

Corradini, Danilo

Correia Soeiro, Maria de

Nazaré

Corsi, Fabio

Cosentino, Marco

Costa, Paulo J

Costantini, Susan

Costello, Derek A.
Coussot, P.

Cozzi, Renata

Cozzolino, Daniel

Crane, Edward J.

Crawford, Philip W.

Creczynski-Pasa, Tania

Crespo, Margarita

Cristobal Lopez, J.

Crupi, Pasquale

Csámpai, Antal

Csuk, René

Cubilla-Rios, Luis

Cubry, Philippe

Cuca Suárez, Luis Enrique

Cuendet, Muriel

Culf, Adrian S.

Cuny, Gregory D.

Curtis, Jonathan M.

Custódio, Luísa

Cvengroš, Ján

Cycoń, Mariusz

Czarnocki, Zbigniew

D'ischia, Marco

Da Costa, Fernando B.

da Silva, A. Moreira

Da Silva, Albérico

Da Silva, Elisiário Tavares

da Silva, Gustavo

da Silva, M. Fátima das G.F.

Dabek-Zlotorzynska, Ewa

D'abrosca, Brigida

Dai, Jungui

Daiber, Andreas

Daly, Norelle

D'Ambrosio, Michele

Dandapani, Sivaraman

Dangles, Olivier

Daniel, Kenyon

Darvesh, Sultan

Darvin, M.E.

Das, Kalyan

David Wang, Hui-Min 
David, Jorge

Davies, Mike

Davis, Matthew C.

De Albuquerque, Julianna F.C.

De Almeida Coelho De Sousa,

Maria João

de Assis, Adriano M.

De Diego, Marta

De Falco, Enrica

de Feo, Vincenzo

De Jesús Ornelas-Paz, José

de Jonge, Ronnie

De Kimpe, Norbert

de Kock, Carmen

De La Fuente, M.A.

De La Herrán, Roberto

de la Hoz, Antonio

De la Mata, F. Javier

De la Monte, Suzanne

de la Torre, Rafael

De La Vega, José Manuel

García

De Leon, Marino

De Marino, Simona

de Medina, F Sánchez

De Oliveira, Anderson R. M.

de Pascual-Teresa, Sonia

De Spirt, Silke

De Tommasi, Nunziatina

De Visser, S.p.

De Vita, Daniela

de Vita, Pasquale

De Wild, Paul. J.

Dealtry, Gill

DeBolt, Seth

Debyser, Zeger

Dechoux, Luc

Deconinck, Eric

Decréau, Richard

Decuypere, Jean-Paul

Decuzzi, Paolo

Dedyukhina, Emiliya G.
Defrancq, Eric

Deharo, Eric

Deivanayagam, Champion

Delgado Olguín, Paul

Delgado, Alejandra

Delgado, Angel

Della Ca, Nicola

Delmastro-Greenwood,

Meghan

Dembinski, Roman

Demchenko, Alexei V.

Demetrescu, I.

Demidova-Rice, Tatiana

Demopoulos, Vassilis J.

Dénès, Fabrice

Denev, Petko

Deng, Lih-Wen

Deng, Lisheng

Dennison, Sarah

Denny, Bill

Denti, Michela A.

Derbré, Séverine

Dernovics, Mihály

D'Errico, Stefano

Desai, Dhimant

Desbois, Andrew

Desbois-Mouthon, Christèle

Desobry, Stéphane

Destaillats, Hugo

Destandau, Emilie

Devred, F.

Dharmarajan, Arunasalam

Dhawan, Deepika

Dhossche, Dirk

Di Donnia, Leonardo Di

Donna

Di Santo, Roberto

Di Venere, Donato

Diana, Marina

Diana, Patrizia

Diao, GuoWang

Diaz, Isabel
Díaz, Isabel

Diaz-Fernandez, Yuri

Díaz-Moreno, Amanda

Consuelo

Díaz-Ricci, Juan C.

Dietrich, Gilles

Diez, David

Dijkwel, Paul

Dimitroff, Charles J.

Dimmock, Jonathan R.

Dinadayalane, Tandabany C.

Ding, Bao-Jian

Ding, Gang

Ding, Jiandong

Ding, Ye

Ding, Yi

Ding, Yuqiang

Ding, Zhong-Tao

Dinjaski, Nina

Djoko, Karrera Y.

Długońska, Henryka

Dobrikov, Georgi

Docheva, Denitsa

Doležal, K.

Domcke, Wolfgang

Domingues, M. Rosário M.

Dominguez, Herminia

Dominguez-Perles, Raul

Dominguez-Ramos, Antonio

Dong, Mingsheng

Dong, Tina T. X.

Donno, D.

Dontas, Ismene A.

D'orazio, John

Dory, Yves L.

dos Santos, Jean Leandro

Doubleday, Charles

Dowling, Kim

Downer, Eric

Downey, Gerard

Pessoa, Otilia D.L.

Drager, Luciano F. 
Dragutan, Valerian

Drašar, Pavel

Drbohlavova, Jana

Dreymüller, Daniela

Drogoudi, Pavlina D.

Drummen, Gregor

Du, Yuguo

Du, Yunfei

Duan, Jin-Ao

Duan, Jinyou

Duan, Kow-jen

Duan, Xiaohui

Duarte, Ana Paula

Dudley, Ed

Dudziak, Diana

Duffy, C. D. P.

Duh, Pin-Der

Dumont, Matthieu

Duncan, James

Durackova, Z.

Durán, Nelson

Durand, Thierry

Durrieu, V.

Dutschk, Victoria

Dutta, Sujoy

Dziedzic, Arkadiusz

Easmon, Johnny

Ebeler, Susan E.

Ebrahimi, Azadeh

Ebrahimi, Samad

Ebrahimzadeh, M.A.

Echevarria, Aurea

Echeverri, Fernando

Ecker, Gerhard F.

Eckert, Gunter P.

Edkins, Adrienne L.

Eduardo-Figueira, Maria

Ee, Pui-Lai Rachel

Egerton, T.A.

Eglin, David

Eiroa, José Luis

Ekuni, Daisuke
El Kaïm, Laurent

El-Achkar, Tarek

Elaissari, Abdelhamid

El-Aneed, Anas

Elhissi, Abdelbary

Eliyan, Faysal Fayez

El-Kareh, Ardith W.

Elkhayat, Ehab S.

Elling, Lothar

Elliot, Carole

Eloff, Jacob

Van Damme, Els J.M.

El-Sayed, Wael A.

El-Seedi, Hesham R.

Emery, Flávio

Enchev, Venelin

Enderlein, Jörg

Engel, Matthias

Enriquez, Rosario

Eparvier, Véronique

Epting, Conrad L.

Erb, U.

Erben, Mauricio F.

Ermakova, Svetlana P.

Erxleben, Andrea

Escuredo, Olga

Espinosa-Aguirre, Jesús Javier

Esposito, Emilio Xavier

Esposito, G.

Esposito, Sergio

Esteban, Maria Ángeles

Esteve-Romero, Josep

Estevinho, Leticia

Estrada-Reyes, Rosa

Estrada-Soto, Samuel

Esumi, Tomoyuki

Evidente, Antonio

Fabian, Walter M.F.

Fabio, Giovanni Di

Fabroni, Simona

Failla, Mark L.

Fairlamb, Ian
Faleiro, Maria L.

Falodun, A

Fan, Chunhai

Fan, Ruiqing

Fan, Wei

Fan, Xue-sen

Fan, Zhijin

Fanali, C.

Fañanas, Francisco J.

Fang, Evandro Fei

Fang, Jia-You

Fang, Jim

Fang, Jinggui

Fang, Ye

Fang, Yulin

Fanning, Kent J.

Fant, Kristina

Fantus, I. George

Fanucci, Gail E.

Farag, Mohamed A.

Farias, Jorge G.

Farid, Chemat

Farinati, Fabio

Farrell, Robert

Farris, Stefano

Farsky, Sandra H.p.

Farver, Ole

Fasano, Elena

Faucheux, Nathalie

Faucitano, Antonio

Faustino, Amparo

Favero, Gabriele

Favi, Gianfranco

Fazio, Alessia

Feás, Xesús

Federico, Alessandro

Fedin, Matvey

Fedorova, Olga S.

Felix Saavedra, Maria José

Feng, Shilan

Feng, Yangbo

Feng, Yibin 
Fenical, William

Fernández-López, Jose

Fernandes-Pedrosa, Matheus

de Freitas

Fernandez, Pablo Lodeiro

Fernandez-Lafuente, Roberto

Fernández-Pérez, Leandro

Fernández-Ponce, $M$. Teresa

Fernando, Pasan

Ferrand, Yann

Ferrari, Erika

Ferrari, Stefania

Ferreira, Isabel

Ferreira, Paula

Ferreira, Vania Maria Moraes

Fett-Neto, Arthur G.

Fiammengo, Roberto

Fiaschi, Tania

Fidele, NTIE KANG

Figadere, Bruno

Filarowski, Aleksander

Filipovic, Milos R.

Filippov, Sergey K.

Fillion, Eric

Finkielsztein, Liliana M.

Finzel, Barry

Fiorentino, Antonio

Fishman, Ayelet

Fitches, Elaine C

Fleisher-Berkovich, Sigal

Flood, Amar

Florea, Bogdan I.

Flores, Pilar

Fock-Bastide, Isabelle

Follmer, Cristian

Fonari, Marina S.

Font, Josep

Fontana, Andreia Cristina

Karklin

Fonteh, Alfred N.

Forlani, Giuseppe

Fornasiero, Paolo
Forni, Alessandra

Forsthuber, Thomas G.

Fossen, Torgils

Foster, Brian C.

Foubelo, Francisco

Fouilland, Eric

Fox, Mark

Fraile, Lorenzo

Frairi, Roberto

Francioso, Antonio

Francis, Heather

Franck, Julien

Frank, Éva

Frappier, Lori

Frau, Roberto

Freije, José M.P.

Freitas, Adilson A.

Freitas, Cleverson D. T.

Freitas, Sandra Borges

Fremd, Carlo

Fribley, Andrew M.

Friesen, Duane

Frija, Luís M. T.

Fritz, Torsten

Froldi, Guglielmina

Frolova, Lilya

Frontera, Patrizia

Froudakis, George E.

Frutos, Maria Jose

$\mathrm{Fu}$, Hua

Fu, Ming-Lai

$\mathrm{Fu}$, Qiang

$\mathrm{Fu}, \mathrm{Tzu}-\mathrm{Fun}$

$\mathrm{Fu}$, Wei

$\mathrm{Fu}$, Yong-Bi

Fujihara, Shinobu

Fujii, Takeshi

Fujimori, Ko

Fujita, Masayuki

Fujiwara, Kenshu

Fukaya, Takayuki

Fukuyama, Takahide
Fukuyama, Tomoki

Fulop, Ferenc

Fuly, André Lopes

Fung, Eileen

Fuqua, Josha

Furuta, Takumi

Fusi, F.

G. Reinecke, Manfred

Gaboriaud, DrNicola

Gagnon, Alexandre

Gahan, Lawrence R.

Gailing, Oliver

Gait, Michael J.

Galdiero, Massimiliano

Galeazzi, Roberta

Galeone, Aldo

Galiano, Silvia

Gali-Muhtasib, Hala U.

Gallo, Alessandra

Gallou, Fabrice

Gallyas, Ferenc

Galvan, Marta

Galvez, Eva M.

Gambaryan, Stepan

Gambin, Yann

Gameiro, Paula

Gámez-Meza, N.

Gan, Kim-Hong

Ganesh, V. Anand

Gangfeng, Ouyang

gannett, Peter

Gao, Feng

Gao, Hao

Gao, Jing

Gao, Jinming

Gao, Kun

Gao, Ruili

Gao, Wei

Gao, Yi Qin

Garau, Alessandra

Garbis, Spiros

García Fernández, José Manuel 
García, Abraham

Garcia, Antonio

García, Hermenegildo

García-Álvarez, Joaquín

García-Beltrán, Olimpo

García-Caballero, Melissa

García-Gonzáles, Rolando

Garcia-Granda, Santiago

García-Sánchez, Miguel A.

García-Villanova, Belén

García-Vivó, Daniel

Gariboldi, Manuela

Garman, Scott C

Garozzo, Domenico

Garro, Marisa S.

Garrote, Gil

Gaspar, Diaz

Gasser, Christoph

Gattuso, Giuseppe

Gaunitz, Frank

Gavicho Uarrota, Virgilio

Gazouli, M.

Gbaguidi, A. Fernand

Ge, Yubin

Geary, Sean

Gebicki, Janusz

Gehrke, Sebastian S.

Geraghty, Dominic P.

Geronikaki, Athina

Gerothanassis, Ioannis P.

Gerschenson, Lía Noemí

Gervay-Hague, Jacquelyn

Gerwick, Cliff

Ghafoor, Abdul

Ghawi, Sameer Khalil

Ghosh-Choudhury, Nandini

Giampieri, Francesca

Giancane, G.

Giannini, Giuseppe

Gibbons, Simon

Gibson, Matthew

Giese, Bernd
Gijzen, Mark

Gikas, E.

Gillies, Elizabeth

Gillinham, Dennis

Gilmer, John F.

Gilmore, TD

Gil-Ramírez, Alicia

Gimenez, Sixto

Gimeno, Miquel

Giner-Casares, Juan J.

Gioiello, Antimo

Giorgi, M.

Giorio, Giovanni

Giovannini, Pier Paolo

Giraldo, Liliana

Girard-Thernier, Corine

Giraudeau, Patrick

Girbés, Tomás

Girousi, Stella

Giudice, Aldo

Gizdavic-Nikolaidis, Marija R.

Glaser, Keith B.

Glatt, H.

Glue, Paul

Gnanasekar, Munirathinam

Gniewosz, Małgorzata

Gobis, K.

Goda, Ahmed E.

Godoy, Sergio Medina

Godt, Adelheid

Gofferjé, G.

Göksu, Süleyman

Goławska, Sylwia

Goldberg, Alfred

Goldstein, Irwin

Goltsov, Alexey

Gomes, Fernanda

Gomes, Sónia

Gómez Di Marco, Perla

Gómez, Irene Crespo

Gómez, Nazely Diban

Gómez, Rafael
Gómez-Caravaca, Ana María

Gómez-Gómez, Lourdes

Gómez-Hens, A

Gómez-Ruiz, Santiago

Gonçalves, G.m.s.

Gonçalves, L.R.B.

Gonçalves, M. Sameiro T.

Gong, Ping

Gong, Xingchu

Gong, Young-dae

González, Concepción C.

Gonzalez, Daniel R

Gonzalez, M. S.

Gonzalez, Mercedes

González-Baró, Ana C.

González-Bello, Concepción

González-Maeso, Javier

González-Mas, M. Carmen

González-Muñiz, Rosario

González-Trujano, Ma. Eva

Gordon, John C.

Gordon, Keith C.

Gordon, Michael

Gorman, Gregory S

Gorrell, M. D.

Gorski, Waldemar

Gorzalczany, S.

Gosmann, Grace

Gostner, Johanna M.

Gotor-Fernández, Vicente

Gou, Shao-Hua

Goulas, Vlasios

Goumont, Régis

Govindarajulu, Rajanikanth

Gowen, Aoife

Goya, Luis

Gozzo, Franco

Grace, Mary

Granica, Sebastian

Grassi, Alfonso

Grassi, Giovanni

Graton, Jérôme 
Grattoni, Alessandro

Gravel, Valerie

Gray, Christopher A.

Gray, Derek G.

Grayer, Renée J.

Grée, René

Greenberg, Robert M.

Greenberger, Joel S.

Greenspan, Phillip

Greenwood, Michael T.

Grgurić-Šipka, Sanja

Grienke, Ulrike

Grigalevicius, Saulius

Grigg, Ronald

Grigoriev, I. P.

Grill, Leonhard

Grimi, Nabil

Grimley, Philip M.

Grindley, T. Bruce

Grose, Julianne H

Gruber, Christian

Gruner, Bohumir

Gruss, Alexandra

Grüttner, Cordula

Grzebisz, Witold

$\mathrm{Gu}$, Jianguo

Gu, Man Bock

$\mathrm{Gu}$, Weikuan

Guddat, Luke W.

Guerin, Marcelo E.

Guerra, Francisco M.

Guetet, Arbi

Gueyrard, David

Guga, Piotr

Guidolin, Diego

Guilhon, Giselle Maria S. P.

Guimaraes Leitão, Gilda

Guisan, Jose Manuel

Gulaboski, Rubin

Gunaje, Jayarama

Guo, Fenghai

Guo, Grace
Guo, Lian-Wang

Guo, Xinwen

Guo, Yuanqiang

Guo, Zhanhu

Guo, Zheng

Gupta, Vinay

Gurzov, Esteban N.

Gust, Ronald

Gutiérrez-Uribe, Janet A.

Ha, Hyun-Joon

Ha, Tae-Yeal

Haba, Hamada

Hackenberg, Michael

Hadjikakou, Sotiris K.

Hadjipavlou-Litina, Dimitra

Haenisch, Sierk

Haertlé, Thomas

Hager, Martin D.

Hagström, ÅK

Hahm, Eun-Ryeong

Hahm, Ki-Baik

Hai, Faisal Ibney

Haider, Norbert

Hak, Sjoerd

Hakamata, Hideki

Halabalaki, Maria

Haleagrahara, Nagaraja

Hall, Edward D.

Haltrich, Dietmar

Hamazaki, Kei

Hamberger, Björn

Hamblin, Michael R.

Hamdane, Djemel

Hamdi, Naceur

Hameed, Shahid

Hammadeh, M.

Han, Bing

Han, Hogyu

Han, Jin

Han, Quan-Bin

Han, Sang-Bae

Han, Shiqing
Han, Yue-Peng

Hanafy, Khalid

Handy, Scott T.

Hanks, Timothy

Hansen, Michael Ryan

Hansen, P.E.

Harbourne, Niamh

Harding, James

Harju, Anni

Harlev, Eli

Haroutounian, Serkos A.

Harrison, William

Hart, Joanne

Hartley, Thomas

Hartmann, Martin

Harvey, Alan

Hasegawa, Makoto

Hasegawa, Urara

Hashimoto, Masaru

Hassan Gilani, S.I., Anwarul

Hassan, Fathi

Hasselmann, Dieter

Hatano, Tsutomu

Haufe, Günter

Hay, Stephen O.

Hayashi, Ken-ichiro

Hayashi, Minoru

Hayashi, Yoshihiko

Hayes, John D.

Hayes, Maria

Haynes, Laura M.

$\mathrm{He}$, Bin

He, Bingfang

$\mathrm{He}$, Hongwu

He, Jian-Uog

He, Jun

He, Liangnian

Heaney, Frances

Hearn, Michael J.

Hebert, Daniel

Hedstrom, Lizbeth

Heemstra, Jennifer M. 
Heger, Michal

Heilemann, Mike

Heinrich, Timo

Heiss, Elke

Helaja, Juho

Helfenstein, Fabrice

Hell, Rüdiger

Hellens, Roger

Hellström, Jarkko

Hendrich, Suzanne

Henle, Thomas

Hennebelle, Thierry

Hensel, A.

Herbert, Cristan

Hermosín-Gutiérrez, Isidro

Hernández Ledesma, Blanca

Hernández, Francisca

Hernandez-Hierro, José Miguel

Herrador, M. Mar

Herraiz, T.

Herranz, Rosario

Herrero-Martínez, José Manuel

Herrmann, Martin

Hesse, Stéphanie

Hiblot, Julien

Hidari, Kazuya I.p.j.

Hideg, Éva

Hideg, Kálmán

Higashi, Shouichi

Hikawa, Hidemasa

Hillard, Elizabeth A.

Himi, Eiko

Hinkula, Jorma

Hipkiss, Alan R.

Hirano, Koji

Hirasawa, Noriyasu

Hirata, Hirokuni

Hiroaki, Hidekazu

Ho, Feng-Ming

Ho, Junming

Ho, Tin-Yun

Ho, Yunn-fang
Höbartner, Claudia

Hocher, Valérie

Hodges, Robert

Hoelder, Swen

Hofer, Tim

Hofmann, Alan F.

Hofmann, Bettina

Hofmann, Ute

Hogg, Philip

Hohlfeld, Thomas

Hohng, Sungchul

Hojo, Keiko

Holder, Alvin A.

Holdt, Hans-Jürgen

Holzgrabe, Ulrike

Hong, Xuechuan

Honma, Tetsuo

Honoré Hansen, Steen

Hood, Matthew A.

Hoogenboom, Bart

Hook, Ingrid L.I.

Horbowicz, M.

Horbowicz, Marcin

Hori, Kanji

Hormi, Osmo

Horobin, Richard W.

Horstkorte, Rüdiger

Hort, Mariana Appel

Hosmane, Ramachandra S.

Hosni, Karim

Hosono, Masahiro

Hosoya, Takashi

Hossain, Mohammad Billal

Hostetler, Heather A.

Ho-Tin-Noé, Benoît

Hou, Chien-Wei

Hou, Tingju

Hou, Tingjun

Hou, Weihong

Houen, Gunnar

Houng, Jer-yiing

Hour, Mann-Jen
Hoving, J. Claire

Howitt, Crispin A.

Hrelia, Silvana

Hsiao, George

Hsiao, Pei-Wen

Hsieh, Pei-wen

Hsieh, Yi-Hsien

Hsieh, Yves

Hsing, Chung-Hsi

Hsu, Hsu

Hsu, Kuo-Chiang

$\mathrm{Hu}$, Deyu

$\mathrm{Hu}, \mathrm{Guoku}$

$\mathrm{Hu}$, Jiang

$\mathrm{Hu}$, MingKuan

$\mathrm{Hu}$, Ping

Hua, Erbing

Hua, Susan

Huang, Beibei

Huang, Chi-Chang

Huang, Chih-Ching

Huang, Hui-Yu

Huang, Jianliang

Huang, Jui-Hsien

Huang, Jun

Huang, Keng-Shiang

Huang, Lin-Fang

Huang, Linjuan

Huang, Tien L.

Huang, Wei-Jan

Huang, Wen-Hsin

Huang, Wenlong

Huang, Xiao-Jun

Huang, Xinchuan

Hubbard, Rod

Huck, Christian

Huczyński, Adam

Hudhomme, Piétrick

Hudson, Robert

Huefner, Antje

Huffaker, Alisa

Hugueney, Philippe 
Huh, Do Sung

Huh, Man Kyu

Huh, Tae-Lin

Hulme, Christopher

Hung, Li-man

Hunsche, Mauricio

Huq, Fazlul

Hurvois, Jean-pierre

Hussein, Ahmed A.

Hussein, Waleed

Huynh, Thuan Minh

Hwang, Bang Yeon

Hwang, Dae Youn

Hwang, Geum-Sook

Hwang, Ki-chul

Hwang, Tsong-Long

Iacobellis, Nicola S.

Iannello, Carmelina

Ichinose, Koji

Ida, Elza Iouko

Igarashi, Kiharu

Igarashi, Miki

Igau, Alain

Iglesias, Marta

Iglesias, Rosario

Iida, Takashi

Iino, Ryota

Ilari, Andrea

Ilic, Nebojsa

Im, Wan-Taek

Imanishi, Akihito

Imberty, Anne

Inagaki, Masaki

Ingram, Susan L.

Inkster, James

Innocentini Mei, Lucia Helena

Inokuchi, Tsutomu

Inoue, Hiroyasu

Insuasty, Braulio

Ionel, Mangalagiu

Iorizzi, Maria

Iorizzo, Massimo
Iossifidou, Eleni

Iriti, Marcelo

Isaad, Jalal

Ishibashi, Masami

Ishihara, Kazuyuki

Ishikura, Minoru

Isidoro, Ciro

Isman, Murray B.

Isobe, Sachiko

Isshiki, Yoshiaki

Ito, Michiho

Ito, Yoichiro

Itoh, Nobuya

Itoh, Tomohiro

Ivanov, Ivo C.

Ivanova, Bojidarka

Ivanova, Elena P.

Iwai, Naoharu

Iwan, Agnieszka

Iwao, Masatomo

Iwasaki, Tomohiro

Iwashina, Tsukasa

Iwata, Tadahisa

J Martín-Rodríguez, Alberto

Jáč, Pavel

Jackson, William F.

Jacob, Claus

Jacobsen, Elisabeth Egholm

Jacquot, Yves

Jaffrezic-Renault, Nicole

Jagerovic, Nadine

Jakob, Ursula

Jakubowski, Joseph A

Jameson, David

Jampilek, Josef

Janetka, James W.

Janfelt, Christian

Jang, C. S.

Jang, Dae Sik

Jang, Hae-Dong

Jang, Hyeung-Jin

Jang, Jinho
Jantas, D.

Jarret, Robert L.

Jasinski, Jerry P.

Je, Jae-young

Jeandet, Philippe

Jefferies, L.K.

Jeffrey, Christopher S.

Jelen, Henryk

Jenks, William

Jensen, Søren Rosendal

Jensen, Svend

Jeon, Byeong Hwa

Jeon, Ju-hong

Jeong, Byeongmoon

Jeong, Ill Yun

Jeong, Lak Shin

Jerković, Igor

Jerz, Gerold

Jeschke, Gunnar

Jesionowski, Teofil

Jeyaseelan, Kandiah

Ji, Bin

Ji, Hai-Feng

Ji, Nai-Yun

Ji, Tengfei

Jia, Li

Jia, Zhenquan

Jiang, De An

Jiang, Guang

Jiang, Guoqiang

Jiang, Lin

Jiang, Ming

Jiang, Yueming

Jiang, Yuyang

Jiao, Wanting

Jimbo, Mitsuru

Jiménez, Ana

Jiménez-Barbero, Jesús

Jimenez-Flores, Rafael

Jimenez-Lopez, Jose

Jin, Tienan

Jindrich, Jindrich 
Jing, Dengwei

Jing, Jing

Jiráček, Jiř́

John A., Beutler

Johnson, Jeremy J.

Johnston, Carl Gordon

Johnston, Carol

Jolivalt, Corinne G.

Jonnala, Surendranadha Reddy

Joo, Choun-Ki

Jordan, Joaquin

Jordão, António M.

Jørgensen, Kåre

Joshi, Tanmaya

Jou, Jwo-Huei

Jovanovic, Jelena D.

Joven, Jorge

Joy, Margarita

Jóźwiak, Krzysztof

Juers, Douglas H.

Juhasz, Bela

Juliá, Luis

Jung, Hyun Ah

Jung, Klaus

Jung, Sang Hun

Junker, Jan Philipp

Jurado, Amália S.

Jurikova, Tunde

Juskowiak, Bernard

Juszczak, Lesław

Juvik, John

K. Deyholos, Michael

Kaczor, Agnieszka

Kaczorek, Ewa

Kad, Neil M.

Kadokawa, Jun-ichi

Kaeko, MUROTA

Kafarski, Pawel

Kai, Guoyin

Kakiyama, Genta

Kakkar, Ashok K.

Kalinin, Stanislav
Kalinin, Vladimir

Kalinowska-Lis, Urszula

Kaliora, Andriana C.

Kallunki, Tuula

Kalska-Szostko, B.

Kaltner, Herbert

Kamei, Junzo

Kamel, Alaa

Kamenecka, Theodore M.

Kaminska, Bozena

Kan, Chi

Kanai, Masashi

Kanan, Yogita

Kanaoka, Masahiro

Kaneco, Satoshi

Kaneda, Kiyotomi

Kaneko, Kimiyuki

Kang, Chulhun

Kang, Hee Eun

Kang, Jong

Kang-Mieler, Jennifer J.

Kanie, Osamu

Kao, Ming-Ching

Kao, Hsien-ming

Kappe, C. Oliver

Karakhanov, Edward

Karimi, Mahsan

Karioti, Anastasia

Karoyan, Philippe

Karpoormath, Rajshekhar

Kaschula, Catherine H.

Kashfi, Khosrow

Kashiwada, Yoshiki

Katagiri, Hiroshi

Katarg, Argyropoulou

Katayama, Hidekazu

Kato, Katsuya

Kato, Koichi

Kato, Tatsuya

Katz, Jonathan I.

Kaufmann, Dieter

Kauhaluoma, Jari
Kawabata, Kyuichi

Kawahara, Teppei

Kazem, Ahmed

Ke, Xuebin

Ke, Yanxiong

Keating, David H.

Keglevich, György

Keijitsu, Keijitsu

Keil, Frerich

Keller, Paul A.

Kellow, NJ

Kelly, Charles

Kennedy, Eileen J.

Kenyon, William J.

Kerwin, Sean M.

Keum, Gyochang

Kevil, Christopher

Keyzers, Rob

Khan, M. Akram

Khan, Nafees

Khiari, Zied

Kiba, Akinori

Kiec-Kononowicz, Katarzyna

Kieffer, Bruno

Kietzmann, Thomas

Kikuzaki, Hiroe

Kilian, Stefanus

Kim, Guncheol

Kim, Hae-Jo

Kim, Harold

Kim, Hee-Sun

Kim, Hyeung-rak

Kim, Jeong-Han

Kim, Jin-hyun

Kim, Jin-Kyung

Kim, Jung-ae

Kim, Keun-Sung

Kim, Ki Hyun

Kim, Kil-Soo

Kim, No Soo

Kim, Sang Kyum

Kim, Sehoon 
Kim, Seung Hyun

Kim, Sun Chang

Kim, Sung Hoon

Kim, Sunghoon

Kim, Yangmee

Kim, Yeong-Shik

Kim, Yeon-Ju

Kim, Yong Hwan

Kim, Young Kyoo

Kim, Yun-bae

Kim, Yuri

Kimura, Hideo

Kimura, Hideto

Kimura, Masanari

King, Charles C.

King, Frank D.

King, Jerry

King, Petra

Kinugawa, S.

Kiparissides, C.

Kireev, Dmitri

Kirsch, Gilbert

Kirsch, Thorsten

Kirst, Herbert A

Kisch, Horst

Kitade, Yukio

Kitagawa, Seiichi

Kjems, Jørgen

Klapötke, Thomas

Klauber-DeMore, Nancy

Kleij, Arjan W.

Klemm, Dieter

Kletsas, Dimitris

Klettner, Alexa

Klinkhamer, Peter G.L.

Kljun, Jakob

Klode, Joachim

Klotz, Lars-Oliver

Klymchenko, Andrey

Knauer, Shirley K.

Knežević, Nikola Ž.

Knölker, Hans-Joachim
Knupp, Gerd

Ko, Kam Ming

Kobayashi, Isao

Kobayashi, Kazuhiro

Kobayashi, Kazuo

Kobayashi, Shizu

Kobayashi, Yuichi

Kobori, Akio

Kobori, Masuko

Kockx, Maaike

Kojima, Akiko

Kojo, Shosuke

Kokotkiewicz, A.

Koksharova, T. V.

Kolehmainen, Erkki

Kolodziejczyk, Joanna

Komaitis, Michael

Komeyama, Kimihiro

Komiyama, Makoto

Kong, In-soo

Kong, Ling-Yi

Kongsted, Jacob

Konig, Helmut

Konrath, Eduardo Luis

Konstantinidis, Stephanos

Kontominas, Michael

Koodali, Ranjit

Koóš, Miroslav

Koppel, Kadri

Korkaya, Hasan

Kornienko, Alexander

Kortner, Trond M.

Kosová, Klára

Kostecka, Małgorzata

Kostogrys, Renata

Koszytkowska-Stawińska,

Mariola

Koufaki, Maria

Kourimska, Lenka

Kouyama, Tsutomu

Kovacs, Lajos

Kowalska, Teresa
Koya, Daisuke

Koźmiński, Wiktor

Kragl, Udo

Krajcsi, Peter

Krajewska, Barbara

Krajka-Kużnik, Violetta

Krása, Josef

Krasnoholovets, Volodymyr

Kraus, Tobias

Krauss, Isaac J.

Krawetz, Roman

Kreis, Wolfgang

Křen, Vladimír

Kretzschmar, Georg

Krishnan, Kannan

Krušić, Melina Kalagasidis

Krysinski, Pawel

Kuan, Yu-Hsiang

Kuang, Hai-Xue

Kubáň, Vlastimil

Kubota, Toshiaki

Kubota, Yoshihiro

Kuca, Kamil

Kuda, Takashi

Kuder, Tomasz

Kudo, Yasusei

Kukovecz, Ákos

Kulma, Anna

Kumamoto, Eiichi

Kumar, Anil

Kumar, Rakesh

Kumar, Satish

Kumirska, Jolanta

Kuno, Toshiya

Kunz, Horst

Kuo, Jen-min

Kuo, Ping-chung

Kuramochi, Kouji

Kurlyandskaya, Galina

Kurz, Thomas

Kusakabe, Ken-ichi

Kveberg, Lise 
Kwasniewski, Misha

Kwiecien, Slawomir

Kwon, Oran

Kwon, Sung Won

Kwon, Youngjoo

La Clair, James

La Rocca, Renato V.

La, Hyun-Joon

Laali, Kenneth K.

Lacaille-Dubois, Marie-Aleth

Lacrampe, Marie France

Ladero, M.

Lagarde, Florence

Lai, Hsi-Mei

Lai, Maoyi

LaJeunesse, Dennis R.

Lakhtin, V.

Lakshmanan, Venkatachalam

Lallès, Jean-Paul

Lam, Henry

Lamberth, Clemens

Lameira, Jerônimo

Lan, Wen-Jian

Lanari, Daniela

Lantto, Perttu

Lapres, John J.

Laquintana, Valentino

Lara-Sanchez, Agustin

Laszlo, Joseph A.

Laufer, Stefan

Lautenschläger, Christian

Lavasanifar, Afsaneh

Lawson, Charlotte

Lay, Horng-Liang

Lazos, Evangelos S.

Lazzarato, Loretta

Leccese, Annamaria

LECLÈRE, Philippe

Lecomte, Philippe

Lee Suan, Chua

Lee, Adam

Lee, Bao-Shiang
Lee, Bruce P.

Lee, Che-Hsin

Lee, Cheng-I

Lee, Choon Young

Lee, Choong Hwan

Lee, Dong-ung

Lee, Dongwon

Lee, Duu-Jong

Lee, Hian Kee

Lee, Hon Man

Lee, Hsin-Chen

Lee, Hsinyu

Lee, Hye Suk

Lee, Jae Kwon

Lee, Jeong-chae

Lee, Jeongmi

Lee, Jin Hyung

Lee, Jin-ching

Lee, Jung Heon

Lee, Jung-Kul

Lee, Jungmin

Lee, Lin-wen

Lee, Min Won

Lee, Moses

Lee, S. Yong

Lee, Sang Gil

Lee, Sang Kook

Lee, Sang-Soo

Lee, Seokjoon

Lee, Seong-Ryong

Lee, Seung Ju

Lee, Shyi-Long

Lee, Sunwoo

Lee, Sun-Young

Lee, $\mathrm{Tu}$

Lee, Tzong-Huei

Lee, Tzong-shyuan

Lee, Won Sup

Lee, Woo Song

Lee, Yong Rok

Lee, Young Sup

Lee, Zang Hee
Leem, Kang-Hyun

Leenders, William P.J.

Legendre, Laurent

Lehle, Karla

Leidinger, Petra

Leitner, Erich

Leivonen, S.K.

Lemkul, Justin A.

Len, Christophe Len

Lenth, Russell V.

Léonel, Eric

Leonetti, Francesco

León-Rivera, Ismael

Lepenies, Bernd

Lépine, Francis

Lepore, Maria

Les, Donald H.

Lesinski, Gregory B.

Lesyk, Roman

Leu, Yann-Lii

Leu, Yu-Ling

Leung, A.W.N.

Leung, Chung-Hang

Leung, Elaine Lai-Han

Leung, Man-kit

Leung, Ping-Chung

Lew, D. Betty

Lezcano, J.M.

Lezot, Frédéric

Li, Donghao

Li, Duo

Li, Fengting

Li, George

Li, Guijie

Li, Hua-Bin

Li, Huige

Li, Jian

Li, Jianming

Li, Jianshu

Li, Jinjun

Li, Juan

$\mathrm{Li}, \mathrm{Ke}$ 


\begin{tabular}{|c|c|c|}
\hline Li, Kefeng & Lin, Carol & Liu, Hsiao-Sheng \\
\hline $\mathrm{Li}, \mathrm{Li}$ & Lin, Chih-Cheng & Liu, Jiang-Yun \\
\hline Li, Nianguang & Lin, Chih-Ching & Liu, Jiaren \\
\hline Li, P.M. & Lin, Chiou-feng & Liu, Jie \\
\hline Li, Ping-Ya & Lin, Chun-Cheng & Liu, Jin \\
\hline Li, Qi & Lin, Chunmian & Liu, Jingbo \\
\hline Li, Shao-Shun & Lin, Hai-shu & Liu, Jun Xi \\
\hline Li, Shiyou & Lin, Hsiang-Ru & Liu, Jun-Jen \\
\hline Li, Shu-Ming & Lin, Hui-Hsuan & Liu, Kexin \\
\hline Li, Wang & Lin, Ivan J.B. & Liu, Lei \\
\hline Li, Wei & Lin, Jing-jer & Liu, Liang \\
\hline Li, Xia & Lin, Jin-yuarn & Liu, Liwang \\
\hline Li, Xiansen & Lin, Jun & Liu, Minghua \\
\hline Li, Xiaobo & Lin, Quankui & Liu, Qiancai \\
\hline Li, Xingshu & Lin, Shyh-Hsiang & Liu, Qing-hui \\
\hline Li, Xingtai & Lin, Wenhan & Liu, Qingsong \\
\hline Li, Xuechen & Lin, Wey-jinq & Liu, Shi J. \\
\hline Li, Yu-Teh & Lin, Yun-lian & Liu, Shing-Hwa \\
\hline Li, Zhiyong & Lin, Zhenjian & Liu, Shi-Xia \\
\hline Li, Zibo & Lin, Zhihua & Liu, Shulin \\
\hline Lian, Jane B. & Lin, Zhixiong & Liu, Wei \\
\hline Lian, Xiao-Yuan & Lincoln, Per & Liu, Xinghai \\
\hline Liang, Chengyu & Lindemann, Peter & Liu, Xinyong \\
\hline Liang, Chia-Hua & Lindequist, Ulrike & Liu, Yang \\
\hline Liang, Hong & Lindhorst, Thisbe & Liu, Ying \\
\hline Liang, Pi-Hui & Lindsey, Keith & Liu, Yonghong \\
\hline Liao, Bo & Link, Andreas & Liu, Yongjun \\
\hline Liao, Jyh-fei & Lioi, Lucia & Liu, Yuanhong \\
\hline Liaw, Chih-Chuang & Lionetto, M. G. & Liu, Yuelian \\
\hline Liby, Karen & Liston, Adrian & Liu, Zai-qun \\
\hline Lichtenstein, Rachel G. & Litinas, Konstantinos E. & Liu, Zhi Long \\
\hline Liebau, Eva & Little, R. Daniel & Liu, Zhongqiu \\
\hline Liebscher, Juergen & Liu, Chang-Xiao & Livrea, Maria Antonia \\
\hline Lila, Mary Ann & Liu, Chong-Huai & Lizard, Gérard \\
\hline Lim, Beong Ou & Liu, Dehua & Lo Faro, Maria Letizia \\
\hline Lim, Chee Wei & Liu, Delong & Lo, Clive \\
\hline Lim, Dongwook & Liu, Dongmin & Lo, Jeng-Fan \\
\hline Lim, Soon Sung & Liu, Fang & Lo, Lee-Chiang Lo \\
\hline Lim, Y.M. & Liu, Feng & Locatelli, Marcello \\
\hline Lima-Brito, José Eduardo & Liu, Gang & Locatelli, Monica \\
\hline Limbach, Hans-Heinrich & Liu, Haining & Loizzo, Monica Rosa \\
\hline Limbach, Michael & Liu, Hai-Yang & Lomberget, Thierry \\
\hline
\end{tabular}


Long, Chao-An

Long, Chunlin

Longo, Pasquale

Longo, Vincenzo

Longobardi, Francesco

López, Concepción

López-Abán, Julio

López-Carballo, Gracia

Lopez-Gallego, Fernando

López-Rodríguez, María L.

Loppi, S.

Lorberboum-Galski, Haya

Lorenzo, E.

Lorkowski, Stefan

Loska, Rafał

Lou, Bih-Show

Lou, Hong Xiang

Lou, Y.J.

Loukovaara, Sirpa

Lovero, Grazia

Lowary, Todd L.

Lowry, Christopher A.

Lozano, Pedro

$\mathrm{Lu}$, Jun

$\mathrm{Lu}, \mathrm{Kui}$

Lu, Ling

Lu, Mei-Chin

$\mathrm{Lu}$, Rong

Lu, Wei

Lu, Yinrong

Lu, Zhaoxin

Lucenti, Elena

Ludwiczuk, Agnieszka

Luesch, Hendrik

Lugemwa, Fulgentius Nelson

Luisi, Renzo

Luna, Jonny E. Duque

Luneau, Dominique

Luo, Cheng

Luo, Huan-Min

Luo, Shenglian

Luo, Xiaoxing
Luo, Yinggang

Luo, Zisheng

Lupiáñez, José A.

Lupidi, Giulio

Lurie, Susan

Lustri, Wilton Rogério

Luzhkov, Victor B.

Lygate, Craig A.

Lykakis, Ioannis

Ma, Bai-ping

Ma, Chaomei

$\mathrm{Ma}$, Chen

Ma, Edmond Dik-Lung

Ma, F.

Ma, Jin Yeul

Ma, Wanhong

Ma, Yu-qiang

Ma, Zhong-Jun

Macaluso, Filippo

MacCallum, Donna

Machon, Denis

MacKenzie, Donald

Mackenzie, Graham

Maćkowski, Sebastian

Macyk, Wojciech

Maddirela, Dilip R.

Madureira, João

Maeda, Isamu

Maeda, Toshinari

Mafu, Sibongile

Magda, Jules J.

Maggi, Filippo

Magid, Abdulmagid Alabdul

Magliozzo, Richard

Magnani, Mauro

Mahdi, Jassem

Mahelka, Václav

Maher, Pamela

Mahiou-Leddet, Valérie

Mahmood, Khalid

Mahrwald, Rainer

Maia, José Guilherme S.
Maioli, Emanuela

Majewsky, Marius

Major, Marian E.

Majumdar, Susruta

Makala, Levi HC

Makino, Toshiaki

Malan, Sarel F.

Malde, Alpesh

Maldonado-Medina, Rosa

Amelia

Malekinejad, H.

Malfa, Stefano La

Malki, Mustapha

Mallapragada, Surya K.

Malmsten, Martin

Maloney, Mark

Mambu, Angele

Mambu, Angèle Lengo

Man, Kwan

Mancheño, José

Mancheño, Olga García

Mancuso, Cesare

Mancuso, Raffaella

Manenti, Stephane

Manganaris, George A

Mangu, Naveen

Mann, Enrique

Mannini, Matteo

Mansoori, G. Ali

Mantell, C.

Manthey, John A.

Mantovani, Alberto

Mantzavinos, Dionissios

Manzhos, Sergei

Mao, Hanbin

Mao, Xiangzhao

Maoz, Ben

Marangoni, Franca

Marasco, Daniela

Marco-Contelles, José

Marie, Emmanuelle

Marik, Jan 
Marimpietri, Danilo

Marin, Jose J. G.

Marin, Luminita

Marín, Sonia

Marinesco, Stéphane

Marino, Angela

Marison, Ian

Marken, Frank

Marković, Zoran

Marmion, Celine J.

Marques, Fernanda

Marques, José C.

Marreilha Dos Santos, Ana

Paula

Marsh, Deborah

Marsich, Eleonora

Martel, Fátima

Martens, Craig C.

Marti, Guillaume

Martí, Sérgio

Martín, A.

Martin, Daniel

Martin, Julia

Martín, Pablo Antonio Lara

Martinelli, Adriano

Martínez, Ana M.

Martinez, Gary V.

Martínez-Díaz, Rafael A.

Martínez-Palou, Rafael

Martinoli, Maria-Grazia

Martin-Ortigosa, Susana

Martins, Filomena

Martins, Luísa M. D. R. S.

Martins, M. R.

Martins, Rosário

Marx, Andreas

Marzouk, Amani M.

Mashtoub, Suzanne

Maskrey, Benjamin H.

Mason, Ronald

Mastino, Antonio

Masu, Hyuma
Masuoka, Noriyoshi

Materska, Małgorzata

Mateus, Nuno

Mathé, Christophe

Mathesius, Ulrike

Matkowski, Adam

Matos, Maria Joao

Matsubara, Kouki

Matsuguchi, Tetsuya

Matsui, Takuya

Matsui, Toshiro

Matsumoto, Kozo

Matsumoto, Yoshimi

Matsunami, Katsuyoshi

Matsuo, Yukiko

Matthaei, Hanno

Matthews, Paul D.

Matyus, Peter

Mavromoustakos, Thomas

Mayer, Christian

Mazorra-Manzano, Miguel A.

Mazutti, Marcio A.

Mazzei, Franco

Mazzoni, I.

McAlpine, Shelli

Mcanulty, Steve

McCallum, Jason L.

McCann, Mark J.

Mccarthy, Peter

McCarty, Owen

Mcconnell, Michelle

Mcdowell, Arlene

McGill, Mitch

Mcginley, John

Mcinnes, Campbell

McMenamin, Paul Gerard

McMullin, David R.

McPhillie, Martin

Medina, Miguel

Medina, Miguel Ángel

Medina, Roxana

Mekhloufi, Ghozlene
Melikov, Kamran

Melino, Sonia

Menaa, Farid

Mena-Rejon, Gonzalo J.

Mendes, Adriano Aguiar

Mendez, Luciana

Méndez-Ardoy, Alejandro

Menendez, Jose Carlos

Menéndez, Pilar

Menezes, José

Meng, Fanhao

Meng, Fenghua

Meng, Qingxiong

Menkissoglu-Spiroudi, Urania

Menna, Marialuisa

Mercader, Andrew G.

Merín, María Gabriela

Merino-Gergichevich, Cristian

Merlini, Lucio

Mesías, Marta

Mesmaeker, Andrée Kirsch-De

Messeguer, Angel

Messori, Luigi

Meyer-Hamme, Gesa

Mezzetti, Bruno

Mi, Man-tian

Miao, Jun-ying

Miceli, Antonio

Michaelakis, Antonios

Micheli, L.

Michellys, Pierre-Yves

Micura, Ronald

Miguel, M. G.

Mikata, Yuji

Mikkonen, Kirsi S.

Miklós, Mézes

Mikstacka, Renata

Mikulski, Damian

Mikusova, Katarina

Milella, Luigi

Milelli, Andrea

Milenkovic, Dragan 
Miller, Anthony B.

Miller, Marshall G.

Milojković-Opsenica, Dušanka

M.

Milstein, David

Mimica-Dukic, Neda

Mimmo, Tanja

Min, Kyunghoon

Min, Sun-joon

Minbiole, Kevin P. C.

Minehan, Thomas G.

Minoura, Mao

Miranda, Katrina

Miranda, L.

Miret-Artés, Salvador

Mirica, Liviu M.

Misaka, Shingen

Mischne, Mirta P.

Misle, Enrique

Mita, Tsuyoshi

Mitakou, Sofia

Mitronova, Gyuzel

Miyabe, Hideto

Miyagishi, Makoto

Miyata, Yoshihiko

Miyazaki, Kouji

Miyazaki, Masaya

Mizuno, Cassia S

Mladěnka, Přemysl

Mlcek, Jiri

Mlinarić-Majerski, Kata

Młochowski, Jacek

Moberg, Christina

Mocanu, G.

Mohan, Tamilselvan

Mohd Esa, Norhaizan

Möhwald, Helmuth

Moineau-Chane-Ching,

Kathleen I.

Mojtahedi, Mohammad M.

Mojžiš, Ján

Mok, Daniel Kam-Wah
Molinari, Alessandra

Molinari, Francesco

Moliner, Manuel

Mollapour, Mehdi

Mollica, Adriano

Molmeret, Maëlle

Molnar, Maja

Molnár, Péter

Monchaud, David

Monforte, Antonio Jose

Monnier, Florian

Monsees, T. K.

Montenegro, Lucia

Montero, Mayte

Montes, Antonio

Montes, Juan

Montes, Vince

Moodley, Brenda

Moo-Huchin, Víctor M.

Moon, Hyung-In

Moon, Jeon-Ok

Moor, Cornelia H. de

Moore, John

Moorthy, N.S. Hari Narayana

Morales, M. P.

Moran, Jeffery $\mathrm{H}$.

Moravcová, Jitka

Morcos, Nabil

Moreno, Diego

Moreno, Silvia

Moretto, Alessandro

Morgan, Kevin

Mori, Naoki

Mori, Yuji

Morikawa, Toshio

Morimura, Shigeru

Moriwaki, Hiroshi

Morreale, Marco

Morris, Gordon A

Morris, Jonathan

Morris, Viola B.

Morschhäuser, Roman
Mosca, Simone

Mossialos, Dimitris

Mostafavi, Mehran

Mott, Ivan

Moujir, L. Moujir

Moutsatsou, P.

Moyano, Albert

Mphahlele, Malose Jack

Muccioli, Giulio G.

Mueller, Jens

Muhle, Claudia

Mukudai, Yoshiki

Mullen, Kathleen M.

Muller, C.j.f.

Muller, Christa

Mullins, Robert F.

Mulvihill, Erin

Muñiz-Salazar, Raquel

Muñoz-Bonilla, Alexandra

Muñoz-Torrero, Diego

Murafuji, Toshihiro

Murakami, Hiroshi

Murakami, Kazuma

Murakami, Manabu

Muramoto, Koji

Murata, Kenji

Murray, Eoin

Murru, Siva

Musa, Musa

Musci, M.

Musco, Giovanna

Musiol, Robert

Muszyńska, Bożena

Muthukrishnan, Subbaratnam

Myung, Chang-seon

Myung, Ja Hye

Myung, Pyung-Keun

Manojlovic, N. T.

$\mathrm{Na}$, Dong Hee

Nadhlala, A. R.

Nag, Arup

Nagai, Kouhei 
Nagai, Ryoji

Nagasawa, Kazuo

Nagy, Istvan

Nagy, Zsombor Kristof

Nakagawa, Kyuya

Nakamura, Itaru

Nakamura, Seiichi

Nakamura, Seikou

Nakamura, Yoshimasa

Nakata, Rieko

Nakatani, Hisayuki

Nalewajko-Sieliwoniuk, Edyta

Namba, Kosuke

Nasri, Rim

Natarajan, Viswanathan

Nathan, Ilana

Navarra, Michele

Navarro, Alfons

Navickiene, Sandro

Nayak, Balunkeswar

Nazaruk, Jolanta

Nazinuddin, B.

Nazzaro, Filomena

Ndip, Edmund

Nebbioso, Marcella

Nechab, Malek

Nedachi, Taku

Negron-Silva, Guillermo E.

Nelson, Peter

Nemykin, Victor

Nerín, C.

Nerli, Bibiana B.

Netto, F. M.

Neugart, Susanne

Neumann, Donna M.

Neumann, Terrence

Neuzil, Jiri

Neves, José Manuel

Neves, M. Graça P.M.S

Newman, David

Newman, Stuart

Nguyen, Phuong
Nguyen, Thanh Binh

Nicasio, M. Carmen

Nicholl, Iain

Nickels, Joseph T

Nicolopoulos, Stavros

Niedzwiedzki, Dariusz M.

Nielsen, Poul

Nihei, Ken-ichi

Nikitina, Viktoriya A.

Nikolic, Dejan

Nikonowicz, Edward P.

Nishi, Mayumi

Nishihara, Yasushi

Nishina, Yuta

Nishitani, Yosuke

Nishiwaki, Nagatoshi

Niu, Xiao-Feng

Nobili, Stefania

Noguer, Thierry

Nomikos, Tzortzis

Nomiya, Takuma

Norambuena, Andrés

Norimoto, Hisayoshi

Norte, Manuel

Nosaka, Yoshio

Notni, Johannes

Nouso, Kazuhiro

Novak, Lajos

Novellino, Ettore

Novo, Salvatore

Nowak, Renata

Nowicka, Anna

Nowicka, Anna M.

Nowicki, Janusz

Ntalli, Nikoletta G.

Nurisso, Alessandra

Obata, Toshihiro

Obenland, David

Oberlies, Nicholas H.

Obreza, Aleš

Ochiya, Takahiro

Ochoa, Garcia
Ochoa-Alejo, Neftali

Oda, Tatsuya

Odell, Luke R.

Odero-Marah, Valerie A

O'Doherty, George

Ogawa, Akiya

Ogawa, Shuichi

Oger, Phil M.

Ogoshi, Sensuke

Ogris, Manfred

Ogungbe, Ifedayo Victor

Oh, Hyuncheol

Oh, Sei-Ryang

Oh, Won Keun

Oh, Won-chun

Ohara, Kazuaki

Ohkohchi, Nobuhiro

Ohno, Shinya

Ohta, Masaya

Ohta, Yoshiji

Oikawa, Daichi

Oikawa, Shinichi

Ojika, Makoto

Ok, Salim

Oka, Masahiko

Oka, Natsuhisa

O'Keefe, Barry R.

Okovytyy, Sergiy

Oku, Hirosuke

Okuda, Masahiro

Olafsdottir, Elin Soffia

Olennikov, D. N.

Oliart-Ros, Rosa María

Oliveira, Cristina

Oliveira, Elisabete

Oliveira, P.A.

Oliveira, Rui

Ollis, David

Olmo, Esther Del

Olsen, Christian

O'malley, Bert, W

Omboni, Stefano 
Ong, Eng Shi

Ono, Masateru

Oottikkal, Shameema

Ooyama, Yousuke

Oppenheimer, Steven B.

Orčić, Dejan

O'Reilly, Elaine

Ormazábal-Toledo, Rodrigo

Orozco, Fabiola Gutierrez

Orrego, Carlos

Ortega, Angel L.

Ortiz, Maria J.

Osada, Kyoichi

Osheroff, Neil

Østergaard, Jesper

Osterloh, Frank

Oszmiański, Jan

Otsuka, Hideaki

Otsuka, Motoyuki

Ott, Ingo

Ott, Maria

Ottmann, Christian

Ottosson, Henrik

Ouali, Armelle

Ovaska, Timo V.

Oyedeji, Adebola

Pace, Elisabetta

Pace, Vittorio

Padilla, Guillermo

Padilla-Martínez, Itzia I.

Paduano, Luigi

Pae, A.N.

Pae, Hyun-Ock

Pagniez, Fabrice

Pakulski, Zbigniew

Palage, Mariana

Palazon, Javier

Palenzuela López, José

Antonio

Palmer, Mike

Palomar, Manuel

Palomo, Claudio
Palou, L.

Palumbo, Gaetano

Pan, Can-ping

Pan, L. K.

Pan, Min-Hsiun

Pan, Qiuhong

Pan, Y. J.

Pan, Yuanjiang

Panacek, A

Panayiotidis, Mihalis I.

Pandanaboina, Sahitya Chetan

Panee, Jun

Paneth, Agata

Panossian, Zehbour

Pant, Deepak

Panza, Luigi

Panzella, Lucia

Papagiannopoulou, Dionysia

Papaioannou, Dionissios

Papaleo, Elena

Papanikolaou, Seraphim

Papenbrock, Jutta

Papetti, Adele

Papy-Garcia, Dulce

Paradiso, Angelo

Paraskevopoulou, Adamantini

Parella, Teodor

Parente, Augusto

Parinandi, Narasimham L.

Park, Il-Kwon

Park, Ji-Yeon

Park, Ki Hun

Park, Kyoung-Chan

Park, Pyo-Jam

Park, Seung-chun

Park, Yong Sun

Parquette, Jon R.

Parra, Roberto

Parveen, Ifat

Paschke, Reinhard

Passarella, Daniele

Pastori, Nadia
Patarrão, Rita

Patel, Mrudula

Pati, Sandra

Paton, Robert S.

Paudel, Liladhar

Paul, Matthew J.

Paull, Jeffrey

Paulmurugan, Ramasamy

Paulsen, Berit Smestad

Pavlidis, Ioannis

Pavón, Francisco Javier

Pawelec, B.

Pearce, A. Norrie

Pecht, Israel

Pecul, Magdalena

Peddabuddi, Gopal

Pediani, John

Pedraza-Chaverri, José

Pedro, Luis G.

Peisert, Heiko

Pelaez, José Walter

Pelicci, Pier G.

Pellei, Maura

Pellequer, Jean-luc

Pelletier, Jerry

Pelosi, Giorgio

Peltzer, M.

Pena Ruiz, T

Pěnčíková, Kristýna

Peng, Ai-Yun

Peng, Jin-Chyau

Peng, Jing

Peng, Junhua

Peng, Ling

Peng, Robert Y.

Peng, Wen-Huang

Peng, Xianghong

Peng, Xiaojun

Peng, Xinhua

Peng, Zhi-Yong

Peppel, Karsten

Pereda-Miranda, Rogelio 
Peregrina, Jesus Manuel

Pereira, Letricia Barbosa

Pérez, Andy J.

Pérez, Silvia

Pérez-Álvarez, J.a.

Perez-Benito, Joaquin F.

Pérez-Correa, J. Ricardo

Perez-Costa, Emma

Pericàs, Miquel A.

Perjési, Pál

Perona, John J.

Perrin, Charles

Perrin, David

Perrow, Kara

Perry, Nigel

Perticone, Francesco

Pertsemlidis, Alexander

Pescitelli, Gennaro

Pestov, A.v.

Péter, Francisc

Peterka, František

Peters, Verena

Petretto, Giacomo L.

Petroni, Katia

Petzer, Jacobus P.

Peuchmaur, Marine

Pfaltz, Andreas

Pfeffer, Frederick M.

Phan, Manh-huong

Pi, Rongbiao

Piaz, Fabrizio Dal

Picariello, Gianluca

Pichert, Annelie

Picking, William D.

Pietras, Alexander

Pietro, Roberta Di

Pietruszka, Jörg

Pignatello, Joseph J

Pii, Youri

Pilc, Andrzej

Pillay, Viness

Piluzza, Giovannella
PINA, Fernando

Pina, João

Pinent, Montserrat

Pinheiro, Carla

Pinheiro, Maria

Pinho, Eva

Pinilla, Clemencia

Pintado, Manuela

Pinto, Diana C. G. A.

Piorkowska, Ewa

Piotrowska, Dorota G.

Piotrowska, Hanna

Pipelier, Muriel

Pirali, Tracey

Pirngruber, Gerhard D.

Pitchiaya, Sethuramasundaram

Pitsikalis, Marinos

Pitucha, Monika

Pitzurra, Lucia

Pizzala, Roberto

Plano, Daniel

Plastina, Pierluigi

Plech, Tomasz

Plourde, Guy L.

Pluen, Alain

Pócsi, István

Pocurull, Eva

Poczai, Péter

Poddel'sky, Andrey I.

Podolak, Irma

Poerschmann, Juergen

Poetsch, Micaela

Pogoń, Krystyna

Pogorelko, Gennady V.

Pohl, Pawel

Poinsot, Verena

Poleshchuk, Oleg

Poleszak, Ewa

Poletto, Matheus

Polson, Shawn W.

Polyak, Steven

Pontiki, Eleni
Popiołek, Łukasz

Poplawska, Magdalena

Popov, S.V.

Popovic, Boris

Poppe, László

Popsavin, Velimir

Porcheddu, Andrea

Porta, Francesca

Porter, John R.

Portilla Salinas, Jaime

Portillo, María Puy

Porzel, Andrea

Pose, Eva

Pöthig, Alexander

Potterat, Olivier

Poulas, Konstantinos

Poupaert, Jacques H.

Pour, Milan

Poyatos, Macrena

Pratsinis, Harris

Pratt, Stephen

Premkumar, Daniel R.

Prenner, Elmar

Prézeau, Laurent

Procopio, Jesús R.

Proescholdt, Martin

Proestos, Charalampos

Pronin, Alexander V.

Prosen, Helena

Protti, Stefano

Prousis, Kyriakos

Psarra, Anna-maria G.

Psomas, George

$\mathrm{Pu}, \mathrm{Xia}$

$\mathrm{Pu}$, Xiaoping

Puglia, Carmelo

Punta, Carlo

Puntoriero, Fausto

Punzo, Francesco

Pusztaszeri, Marc

Pyrzynska, Krystyna

Pytkowska, Katarzyna 
Qi, Xin

Qian, Bing-Jun

Qian, Hong

Qiao, Chunhua

Qin, Bo

Qin, Chuanguang

Qin, Hailin

Qin, Lei

Qin, Lu-Ping

Qin, Xuemei

Qiu, Feng

Qiu, Hongdeng

Qiu, Jianhui

Qiu, Ming-hua

Quadrelli, Paolo

Quan, Taihao

Quan, Zheshan

Quinn, Ronald

Quintard, Jean-Paul

Rablen, Paul R.

Racchi, M. L.

Racz, I.

Raczyńska, E. D.

Radi, Marco

Radominska-Pandya, Anna

Raederstorff, Daniel

Raffa, Vittoria

Raffaele, Frazzi

Raffaelli, Nadia

Ragaini, Fabio

Ragno, Gaetano

Ragno, Rino

Rai, Dilip K.

Raillard, Cécile

Rak, Janusz

Rakanovic-Todic, Maida

Rakitin, Oleg

Rakotondraibe, L.H.

Ralston, Katherine

Ramalhosa, Elsa

Ramesh, Rajagopal

Ramig, Keith
Ramos, Márcio Viana

Ramsay, Rona R.

Ramsh, S. M.

Rana, D.

Ranieri, Annamaria

Rao, P. N. Praveen

Rao, Zhiming

Rapisarda, Paolo

Rapposelli, Simona

Rasmussen, Seth C.

Rasulev, Bakhtiyor

Rau, Sven

Rauhut, Guntram

Rautio, Jarkko

Ravu, Ranga Rao

Rawel, H. M.

Rawlings, Neil D.

Rawls, Henry

Ray, Ratna

Reboul, Vincent

Reddy, M. V. Ramana

Redfors, Björn

Rehman, Junaid U.

Reible, Danny

Reich, Adam

Reigosa, Manuel J

Reiner, Martin

Reiser, Oliver

Reisman, David

Reisman, Scott A.

Remelli, Maurizio

Remião, Fernando

Ren, Gui-Xing

Ren, Jian-guo

Ren, S. P.

Renault, Hugues

Renčiuk, Daniel

Rendueles, Olaya

Renner, Ulrich

Reta, Mario

Revilla, Eugenio

Reyes, Fernando
Reynal, Anna

Reynisson, Jóhannes

Rho, Jung-Rae

Ribeiro, Bernardo Dias

Ricca, Ezio

Riccardi, Carlo

Ricci, Alfredo

Richomme, Pascal

Richter, Wito

Rico, Milagros

Riedmaier, Irmgard

Riela, Serena

Rigano, Daniela

Rijo, Patrícia

Rimoldi, Isabella

Rinaldi, A. C.

Rinaldi, Alessandra

Rinaudo, Marguerite

Rios, Yolanda

Riou, Laurent M.

Riu-Aumatell, Montserrat

Rivera Sánchez, Gildardo

Rivière, Céline

Rizzolo, A.

Rizzuti, Bruno

Roberge, Michel

Roberti, Marinella

Robina, Inmaculada

Robinson, Dominic J.

Rocamora, Mercè

Roca-Sanjuán, Daniel

Rocheleau, Jonathan V.

Rodilla, Jesus M.

Rodiño, Paula

Rodolfo, Carlo

Rodrigues, Ana Lúcia S.

Rodrigues, Rafael Costa

Rodrigues, S

Rodriguez, Laura

Rodríguez-Gonzalo,

Encarnación 
Rodriguez-Velamazan, Jose

Alberto

Roes, Marilise Le

Roesler, R.

Rogers, Natasha

Rohn, Sascha

Roland, Sylvain

Roleira, Fernanda M.F.

Rolfe, Barbara E.

Rolfe, K. J.

Rolim Medeiros, Jand-venes

Rolle, Luca

Romagnoli, Romeo

Romanelli, Gustavo P.

Romero, M. Angeles

Romero-Castro, Aurelio

Rondeau, Philippe

Rong, Jianhui

Ros, M. Blanca

Rosa, Luis

Rosati, Ornelio

Rosato, Antonio

Roseiro, Luísa B.

Rosenberger, Thad A.

Roskoski, Robert

Ross, Carolyn F.

Ross, Samir

Rosselli, Sergio

Rossi, Elisabetta

Rossius, Vassilios

Rothenberg, Gadi

Rothstein, Jonathan P.

Roubelakis, Emmanuel

Rouseff, Russell L.

Routray, Winny

Rowley, Christopher

Rozen, Shlomo

Ruan, Han-Li

Rubert, Josep

Rubis, Blazej

Rudolf, Emil

Rudzitis-Auth, Jeannette
Ruggerone, Paolo

Ruijter, Dr E.

Ruijter, Eelco

Ruiz, José

Ruiz-Azuara, Lena

Russo, Gian Luigi

Russo, Mario Vincenzo

Russowski, Dennis

Russu, Wade A.

Ruud, Kenneth

Ryan, Geraldine D.

Ryu, Jae-Ha

Ryu, Shi Yong

Saaby, Lasse

Saavedra, Lucila

Sabila, Paul Sali

Sabio, Guadalupe

Sadekar, Shraddha

Safe, Stephen

Sagredo, Onintza

Saha, Achinto

Saito, Shinichi

Saito, Takao

Sakai, Ryuichi

Sakakibara, Hiroyuki

Sakamoto, Naoya

Sakwe, Amos

Salas, Cristián

Salas, Juan M.

Salatino, Antonio

Saldívar-Guerra, Enrique

Saleh, Mahmoud A.

Salgado, Josefa

Salgueiro, Ligia

Salinas, Rosario

Saliu, Francesco

Salomon, Claudio J

Salter, Russell

Salvalaglio, Matteo

Samaroo, Diana

Šamec, Dunja

Samir, Bondock
Samson, Andre L.

Samuelsen, Anne Berit C.

Sánchez, Gerardo

Sánchez-Campos, Sonia

Sánchez-Fidalgo, Susana

Sanchez-Moreiras, Adela

Sánchez-Moreno, Concepción

Sánchez-Palomo, Eva

Sanchez-Sanz, Goar

Sanchiz, Joaquin

Sandwick, Roger

Sang, Shengming

Sanjust, Enrico

Sanny, Charles G.

Sano, Shigeki

Santilio, A.

Santillan, Rosa

Santini, Carlo

Santini, Daniele

Santos, Maria M. M.

Santos, Sónia A. O.

Sanz, Maria

Sanzani, Simona Marianna

Sârbu, Costel

Sassi, Paola

Sasson, Yoel

Sato, F.

Sato, Kenji

Sato, Yuichiro

Saturnino, Carmela

Sautreau, Asmita

Sava, Gianni

Savić, Snežana

Savoie, Jean-Michel

Saxena, Sunil

Scarso, Alessandro

Scendo, Mieczyslaw

Schapiro, Igor

Scheerens, Joseph C

Scheiner, Steve

Schepetkin, IA

Schiaffino, M. Romina 
Schinzer, Dieter

Schiraldi, Chiari

Schirawski, Jan

Schirrmacher, Ralf

Schlaad, Helmut

Schlierf, Michael

Schmeda-Hirschmann,

Guillermo

Schmelz, Eric A.

Schmid, Peter

Schmidt, Heinar

Schmidt, Jürgen

Schmidt, Marie

Schmidt, Thomas J.

Schmitzer, Andreea R.

Schneider, Bernd

Schneider, Gyula

Schnermann, Martin

Schnitzler, Egon

Schols, Dominique

Scholz, Bettina

Schottenberger, Herwig

Schubert, Mario

Schulz, Benjamin L.

Schulz, R.

Schulze, Benjamin

Schwalbe, Harald

Schwan, Adrian L.

Schwartz, Steven D.

Schwarz, Quenten

Schweizer, Frank

Scilimati, Antonio

Scott, Jason

Scotti, Marcus

Scozzafava, Andrea

Scriba, Gerhard

Seca, Ana

Sedlak, Milos

Seehase, Sophie

Seela, Frank

Seely, Katerin

Seidel, Véronique
Seidler, Daniela G.

Seifert, Karlheinz

Sekhar, J. A.

Selbo, PÅl K.

Selicharová, Irena

Selvaggini, Roberto

Semenov, Victor

Sempere, Lorenzo F.

Senanayake, Thulani H.

Senba, Masachika

Senchina, David S.

Sendker, Jandirk

Seneviratne, Chaminda J.

Seong, Yeon Hee

Serpersu, Engin H.

Serralheiro, Maria Luísa M.

Serrano, María

Setaka, Wataru

Sethi, Gautam

Settanni, L.

Setzer, William

Sevilla, María-Ángeles

Sevilla, Michael

Seyedi, Neda

Sforcin, José Maurício

Shah, Dilip

Shah, Rashmi R.

Shaikh, Mohammed Abid

Shalaby, T.

Shalliker, R.A.

Shan, Wei-Guang

Shao, Yawei

Sharma, Amar Deep

Sharma, Arun K

Sharma, Virender K.

Shaw, Arthur Y.

She, Zhigang

Sheldon, Roger

Shen, Haoyu

Shen, Jianfu

Shen, Jiangang

Shen, Jinfeng
Shen, Qi

Shen, Qiang

Shen, Yingjia

Shen, Yue-Mao

Shenderovich, Ilya G.

Sheng, Baiyang

Sherwin, Catherine M. T.

Sheu, Jyh-Horng

Shi, Daqing

Shi, Dayong

Shi, Donglu

Shi, Jie-hua

Shi, Weiming

Shi, Xiaodong

Shi, Yongquan

Shibahara, Fumitoshi

Shibata, Norio

Shigemori, Hideyuki

Shih, Mei-Hsiu

Shimada, Tsutomu

Shimizu, Kuniyoshi

Shimizu, Linda

Shimoda, Hiroshi

Shin, Hyung Shik

Shin, Injae

Shin, Seokmin

Shin, Sung Chul

Shipley, Paul

Shirahata, Takashi

Shmatov, M. L.

Shoyama, Yukihiro

Shukla, Shruti

Siddiqui, Rafat

Siewert, Bianka

Sikora, Ewa

Silaghi-Dumitrescu, Radu

Sillanpaa, Mika

Silva, Ana Rosa

Silva, Fátima Regina Mena

Barreto

Silva, J. P. Sousa e

Silva, Luís R. 
Silva, María Fernanda

Silva, Sandra D.

Silván, Jose M.

Silver, Lynn

Silvério, Flaviano Oliveira

Silvestri, Elena

Simal-Gándara, Jesús

Simirgiotis, Mario

Simmler, Charlotte

Simões, Maria Fátima

Simon, Hans-uwe

Simoneau, Philippe

Simonsen, Henrik

Simpson, Bradley S.

Sinclair, Andrew

Sindler-Kulyk, Marija

Singer, Robert D.

Singh, I.

Singh, Kumud K.

Singh, Vijay K.

Singleton, Daniel A

Siniscalco, Dario

Siren, Heli

Sirover, Michael

Sissi, Claudia

Siu, Parco M.

Sivamani, Raja K

Siwek, Agata

Skalicka-Woźniak, Krystyna

Skoda-Földes, Rita

Sköld, Christian

Skrobik, Yoanna

Slavov, Anton

Sławiński, Jarosław

Sliva, Daniel

Sloan, Kenneth B.

Słoczyńska, Karolina

Slominski, Andrzej

Slusarenko, Alan J.

Smagghe, Guy

Šmejkal, Karel

Smietana, Michael
Smith, Graham

Smith, Keith

Smith, Martyn T.

Smith, Paul A.

Smith, Robert E.

Smoglia, James M.

Smyth, Thomas J.

Snow, Nicholas

Snyder, James P.

Sobarzo-Sánchez, Eduardo

Sobczak, Marcin

Sobotik, Pavel

Södergård, Anders

Soengas, Raquel

Sokovic, Marina

Solans-Monfort, Xavier

Soldan, Samantha

Solioz, Marc

Soll, Dieter

Solla, Humberto

Somoza, álvaro

Song, Fengrui

Song, Yang-heon

Song, Zhihong

Sopade, Peter A.

Sorci, Leonardo

Soreq, Hermona

Soriano-García, Manuel

Sorice, Maurizio

Sorokin, A.b.

Sorrenti, Valeria

Sosa, Alfonso T. García

Sosa, Silvio

Sosnovskikh, V. Ya.

Sosnowska, Anita

Soto, Carmen

Soubhye, Jalal

Sousa, Hugo

Sousa, M.C.

Sousa, Sérgio Filipe

Souza-Fagundes, Elaine M.

Sovage, Imre
Sowers, Lawrence C.

Sozzi, Gabriella

Spadavecchia, Jolanda

Spampinato, Santi

Sparagano, Olivier AE

Spatafora, Carmela

Speeckaert, Marijn

Spendier, Kathrin

Spitalsky, Zdenko

Spiteller, Michael

Spittau, Björn

Sporn, Michael B.

Spring, David

Spring, Otmar

Squina, F.M.

Srivenugopal, Kalkunte

Stadler, Marc

Staerk, Dan

Staiger, Christopher J.

Stajic, Mirjana

Stamatatos, Theocharis C.

Stamatis, Haralambos

Stanković, Milan S.

Stanley, Joanna L.

Stanovnik, Branko

Stavber, Stojan

Stec, Jozef

Stefanovic, Branko

Steindel, Mário

Steinmann, Eike

Stephanidou-Stephanatou, Julia

Stephen Cho Wing, Sze

Stępkowski, Tomasz M.

Stevanato, Roberto

Steven, Sebastian

Stevenson, Bradley

Stevenson, Julian

Stewart, Michael

Stiehler, Maik

Stiff-Roberts, Adrienne D

Stochmal, Anna

Stoika, R.S. 
Stojakowska, Anna

Stojanovic, Ivana

Stone, Koni

Stone, Michael

Stoppacher, Norbert

Stortz, Carlos A.

Strand, Daniel

Stranger, Rob

Streit, Wolfgang R.

Strekowski, Lucjan

Struga, Marta

$\mathrm{Su}$, Nan-Wei

$\mathrm{Su}, \mathrm{Shu}-\mathrm{Jem}$

$\mathrm{Su}$, Wen-Da

Su, Zhushang

Su, Zi-Ren

Suárez, Juan

Sugahara, Takuya

Sugai, Takeshi

Sugawara, Akira

Sugiura, Yoshimasa

Sugumaran, Manickam

Suh, Hyung Joo

Suib, Steven L.

Sukumar, N.

Sulová, Zdenka

Sülzmann, Holger

Sumarah, Mark

Sun, Bai-Wang

Sun, Changhao

Sun, Cheng

Sun, Grace

Sun, Hongzhe

Sun, Hui

Sun, Runcang

Sun, Yongxue

Sung, Jung-Suk

Sung, Ping-Jyun

Sun-Waterhouse, Dongxiao

Süss-Fink, Georg

Sussman, Mark A.

Sutherland, Hamish S.
Sutherland, J.B.

Sutter, E.M.M.

Suzuki, Hideyuki

Suzuki, Hiromu

Suzuki, Masahito

Suzuki, Tadashi

Suzuki, Takafumi

Suzuki, Takahiro

Suzuki, Takanori

Suzuki, Takuya

Svensson, Sara

Syed, Moinuddin

Szczepanik, Beata

Szekeres, Thomas

Szopa, Agnieszka

Sztanke, Małgorzata

Tabata, Keiichi

Tacconelli, Stefania

Tachikawa, Masanori

Tacke, Matthias

Taddei, Maurizio

Tadic, Vanja M.

Taghibiglou, Changiz

Taglialatela-Scafati, Orazio

Tagliazucchi, Davide

Tago, Megumi

Tai, Wanyi

Taillefumier, C.

Taira, Junsei

Takada, Yoshikazu

Takagi, Masatoshi

Takano, Toshiyuki

Takao, Ken-ichi

Takasu, Kiyosei

Takayanagi, Toshiyuki

Takeda, Makio

Takeda, Youhei

Takemori, Hiroshi

Takemura, Genzou

Taki, Masumi

Takii, Takemasa

Talbot, Nicholas J.
Talele, Tanaji T

Tam, Kam Chiu

Tamariz, Joaquín

Tamiaki, Hitoshi

Tan, Chin Ping

Tan, Dun-Xian

Tan, Jian-wen

Tanaka, Akane

Tanaka, Hitoshi

Tanaka, Shunsuke

Tang, Dingqin

Tang, Feng-Yao

Tang, Tao

Tang, Wei

Tang, Xing

Tang, Yiqun

Tang, Yuping

Tanga, Mary

Taniguhci, Takaaki

Tantillo, Dean

Taran, Frédéric

Tarozzi, Andrea

Tarr, Matthew A.

Tateishi-Karimata, Hisae

Tattini, Massimiliano

Taurin, Sebastien

Taveira, Marcos

Taylor, Michelle

Taylor, Peter

Taylor, T. Matthew

Teixeira, Cátia

Teixeira, Róbson

Teixidor, Francesc

Teng, Yuanwen

Teppema, Luc J.

Terekhova, Irina V.

Tesarová, Eva

Teschke, Rolf

Tesmer, John J. G.

Thadani, Udho

Thakur, Mayank

Thèvenod, Frank 
Thews, Oliver

Thirunavukkarasu, Mahesh

Thomas, Helen E.

Thomas, Raymond H.

Thompson, Paul R.

Thomson, Leonor

Thoo-Lin, Paul Kong

Thordarson, Pall

Tian, Ping

Timopheev, A.A.

Timoshenko, Alexander V.

Timperio, Anna Maria

Timsit, Youri

Titanyuk, Igor D.

Tobacman, Joanne K.

Tocchetti, Carlo G.

Todorovic, Tamara

Toegel, Stefan

Toida, Toshihiko

Tokuda, Gaku

Tokuda, Harukuni

Toma, Henrique E.

Tomečková, Vladimíra

Tommonaro, Giuseppina

Tonelli, Alan E.

Tong, Lingying

Tonnabel, Jeanne

Toohey, John I.

Topham, Paul D.

Topuzova-Hristova, Tanya

Tori, Motoo

Toropov, Andrey A

Torras, J.

Torre, Maria

Torre, Maria Luisa

Torreggiani, Armida

Torrens, Francisco

Toyomasu, Tomonobu

Toyooka, Naoki

Trabace, Luigia

Trabbic, Christopher

Tramontini, Sara
Tran, Clara T. H.

Trindade, Alexandre

Trindade, Reginaldo Almeida

da

Trivedi, Prabodh Kumar

Trougakos, Ioannis

Trouillas, Patrick

Trudell, Mark

Tsai, C.Y.

Tsai, Cheng-Fang

Tsai, Shau-Wei

Tselios, Theodore

Tseng, Chao-neng

Tsenkova, Roumiana

Tsim, Karl W.K

Tsipis, Athanassios C.

Tsubaki, Shuntaro

Tsuge, Takeharu

Tsukamoto, Koji

Tsukiyama-Koharaemail,

Kyoko

Tsunemasa, Noritaka

Tsutsui, Shigeyuki

Tu, Pengfei

Tuberoso, Carlo

Tulla-Puche, Judit

Tuñón, Iñaki

Turck, Natacha

Turel, Iztok

Turks, Māris

Turło, Jadwiga

Tuttolomondo, M.E.

Tyler, Betty

Tzeli, Demeter

Tzia, C.

Ubiali, Daniela

Uchi, Hiroshi

Uchida, Yoshikazu

Uddin, Md. Jashim

Uddin, Zakir

Ueda, Mitsuhiro

Ueda, Natsuo
Ueda-Nakamura, Tânia

Ueno, Makoto

Uhrin, Dusan

Uivarosi, Valentina

Ukic, Sime

Ulanski, Piotr

Ulber, Roland

Ulrih, Nataša Poklar

Uludağ, Hasan

Unkefer, Clifford J.

Upadhyay, Atul

Urbano-Cuadrado, Manuel

Urbieta, María Tabernero

Uriarte, Eugenio

Usami, Yoshihide

Uto, Yoshikazu

Uyama, Hiroshi

Vacek, Jan

Vale, Nuno

Valentao, Patrícia

Valero, Francisco

Valero, M.

Valgimigli, L.

Vallés, Cristina

Valmikanathan, O.P.

Van Aerschot, Arthur

Van Calenbergh, Serge

Van De Venter, Maryna

Van den Ende, Wim

van der Meijden, Maarten

Van Der Veken, Pieter

Van Griensven, Leo J.L.D

Van Hook, M.J.

Van Kampen, Jackalina M.

van Thor, Jasper J.

Vanden Eynde, Jean Jacques

Vanderlei de Souza, Maria de

Fátima

Vankempen, Leon

Vankova, Radomira

Váradi, Györgyi

Varela-Ramirez, Armando 
Varese, Giovanna Cristina

Vargas-Bello-Pérez, E.

Vargas-Rodriguez, Yalma L.

Varrot, Annabelle

Varvounis, George

Vasconcelos, Helena

Vasilakoglou, Ioannis

Vasile, Bogdan Stefan

Vautard, Frederic

Vávrová, Kateřina

Vaz, Deisi Altmajer

Vaziri, Nosratola D.

Vázquez-Flota, Felipe

Vecchione, Carmine

Vegvari, Akos

Veiga-Junior, Valdir

Velasco, Pablo

Velez, Zélia

Veneziani, Rodrigo

Venkataraman, Shrinivas

Venturella, Giuseppe

Venukadasula, Phanindra

Veres, Balazs

Verhelst, Steven

Very, Thibaut

Vesuna, Farhad

Vetter, Walter

Vetvicka, Vaclav

Vidanarachchi, Janak K.

Vidossich, Pietro

Vieira, Mónica

Vigier, Karine

Vijver, Pieter Van de

Vila, José Manuel

Vilar, Vítor J.P.

Vilela, Alice

Villani, Claudio

Villegas, Alejandro Madrid

Virkel, Guillermo

Virlogeux-Payant, Isabelle

Visioli, Francesco

Vistnes, Maria
Viuda-Martos, Manuel

Vivas-Reyes, Ricardo

Vo-Dinh, Tuan

Vomiero, Alberto

Von Knethen, Andreas

Vorsa, Nicholi

Voss, Jürgen

Voutquenne, Laurence

Vrchotová, Naděžda

Vuong, Quan V.

Wagner, Caroline

Wagner, Gergard

Wahl, M. A.

Waksmundzka-Hajnos,

Monika

Walker, Anne-sophie

Wallis, Russell

Walorczyk, Stanisław

Wan, David CC

Wan, Jian-bo

Wanderley, Almir G.

Wang, Chi Chiu

Wang, Ching-Chiung

Wang, Chin-Kun

Wang, Cunde

Wang, Deyun

Wang, Fei

Wang, Guangshun

Wang, Hexiang

Wang, Hongbing

Wang, Hongjun

Wang, Horng-dar

Wang, Jeh-jeng

Wang, Jiadao

Wang, jianguo

Wang, Jianji

Wang, Jianping

Wang, Junzhi

Wang, Kai

Wang, Ke-Rang

Wang, Lai-xi

Wang, Minghua
Wang, Peng George

Wang, Qiangbin

Wang, Qun

Wang, Rui

Wang, Shaoyun

Wang, Sheng-Yang

Wang, Shun-Yi

Wang, Shusheng

Wang, Thomas T. Y.

Wang, Wei-hsien

Wang, Wei-Ning

Wang, Xia

Wang, Xiao-Bing

Wang, Xiaoying

Wang, Xiaoyong

Wang, Xinluan

Wang, Xuedong

Wang, Xumei

Wang, Yonghua

Wang, Youwei

Wang, Yuanpeng

Wang, Yue-Hu

Wang, Yuqi

Wang, Zhao-Yang

Wang, Zhimin

Wang, Zhiwen

Wang, Zhiyong

Wängler, Björn

Want, Elizabeth J.

Ward, Dale E.

Wardrop, Duncan J.

Warmuth, Ralf

Watanabe, Eiki

Watanabe, Kazuki

Watanabe, M

Watanabe, Naoki

Watanabe, Yasuharu

Waterschoot, Jasmien

Webb, Thomas

Webber, Ralf

Weber, Bernd

Weber, Heike 
Weber-Ban, Eilika

Wei, Na

Wei, Xiao-Yi

Wei, Zhiyong

Weigand, Wolfgang

Weingart, Georg

Weiss, Clemens K.

Weiss, H. Markus

Welch, K. D.

Weldon, Sinead

Wells, Shawn

Wen, Kuo-Ching

Wenthold, Paul G

Wenzel, Anna G.

Werner, Maria

Werz, Daniel

Wesolowski, Marek

West, James D.

Westerlind, Ulrika

Westman, Gunnar

Weston, Leslie

Westwell, Andrew D.

Wherland, Scot

Whitlock, Christine

Whitman, Malcolm

Whyte, Claire

Wichapong, Kanin

Wicker, Louise

Wiczk, Wiesław

Wiczkowski, Wieslaw

Wieczorek, Piotr

Wielgomas, Bartosz

Wijkander, Jonny

Wilden, Jonathan

Williams, David M.

Williams, Noelle S.

Williams, Spencer

Williamson, Gary

Willmore, William G.

Wills, Martin

Wilson, James N.

Wilson, Lee D.
Winckler, Thomas

Windey, Karen

Wink, Michael

Winnicka, Katarzyna

Winssinger, Nicolas

Winterhalter, Mathias

Wise, Steven G

Wishart, Jane

Wiskur, Sheryl L.

Wittmann, Jürgen

Wittrant, Yohann

Wnuk, Stan

Woelwer-Rieck, Ursula

Wójciak-Kosior, Magdalena

Wojdylo, Aneta

Wojnárovits, Laszlo

Wolan, Dennis W.

Wolska, Krystyna I.

Wong, C K

Wong, Danny K. Y.

Wong, Fung Fuh

Wong, Jonathan Woon Chung

Wong, Ka-Leung

Wong, Kenneth K. Y.

Wong, Man-Sau

Woo, Mi Hee

Wood, W. Gibson

Workentin, Mark S.

Wormley, Jr., Floyd L.

Wöstemeyer, Johannes

Wright, Clyde J.

Wright, Dennis

Wright, Louwrance

Wrolstad, Ronald E.

$\mathrm{Wu}, \mathrm{Bin}$

Wu, Chieh-Hsi

$\mathrm{Wu}$, Chin-Chung

Wu, Chi-Rei

Wu, Chung-Yi

Wu, Ho-Shing

$\mathrm{Wu}$, Huayue

$\mathrm{Wu}$, Jianyong
$\mathrm{Wu}$, Jyh-Horng

$\mathrm{Wu}, \mathrm{Li}$-chen

$\mathrm{Wu}$, Ming-chang

Wu, Qi

$\mathrm{Wu}$, Ruilian

$\mathrm{Wu}$, Shihua

$\mathrm{Wu}$, Tao

Wu, Tzu-Hua

$\mathrm{Wu}$, Xiao-Feng

$\mathrm{Wu}$, Yisheng

$\mathrm{Wu}, \mathrm{Yu}-\mathrm{Tse}$

$\mathrm{Wu}$, Yuyang

$\mathrm{Wu}$, Zhaofei

Wube, Abraham Abebe

Wuest, Frank

Wujec, Monika

Wybraniec, Slawomir

Wysocki, Laura

Wyzgoski, Faith J.

Xavier, Nuno Manuel

Xia, Wenshui

Xian, Ming

Xiang, Lan

Xiao, Dong-Guang

Xiao, Sulong

Xiao, Yongsheng

Xiao, Zhousheng

Xie, Hua

Xie, Qian

Xie, Xiaoliang Sunney

Xie, Yong-Mei

Xin, Zhihong

Xiong, Bing

Xiong, Gaofeng

Yan, Xiongo

$\mathrm{Xu}$, Baoshan

$\mathrm{Xu}$, Changjie

$\mathrm{Xu}$, Feng

$\mathrm{Xu}$, Guobao

$\mathrm{Xu}$, Peng-Fei

$\mathrm{Xu}$, Qiong-ming

$\mathrm{Xu}$, Shichao 
$\mathrm{Xu}$, Weiwei

$\mathrm{Xu}$, Wen-Fang

$\mathrm{Xu}$, Xiao-Jun

$\mathrm{Xu}$, Xudong

$\mathrm{Xu}$, Xueming

$\mathrm{Xu}$, Yan

$\mathrm{Xu}, \mathrm{Yi}$-Jun

$\mathrm{Xu}$, Zheng-Hong

Xu, Zhimin

Xue, Ming

Yaegaki, Ken

Yagi, Akira

Yagüe, Ernesto

Yahara, Shoji

Yamada, Shinji

Yamada, Shuhei

Yamagishi, Nobuyuki

Yamaki, Koji

Yamamoto, Hisashi

Yamanaka, Masamichi

Yamasaki, Sho

Yamashita, Fumiyoshi

Yamato, Takehiko

Yamauchi, Satoshi

Yan, Chao-Guo

Yan, Fei

Yan, Jizhong

Yan, Rulong

Yan, Xiufeng

Yan, Yunjun

Yan, Yun-jun

Yanez, Claudia

Yang, Chao-Hsun

Yang, Chunping

Yang, Depo

Yang, Ding-Yah

Yang, Guang-Fu

Yang, Haw

Yang, Hongjun

Yang, Hyun Ok

Yang, Li

Yang, Ling
Yang, Liqun

Yang, Liuqing

Yang, Paul

Yang, Qing

Yang, Shun-Fa

Yang, Song

Yang, Wei

Yang, Wei-hsiung

Yang, Wen-bin

Yang, Xian-wen

Yang, Xiuwei

Yang, Yong-hua

Yang, Yu-Chiao

Yang, Yu-Rong

Yang, Yushe

Yang, Z.-Q.

Yang, Zhijun

Yao, Guangmin

Yao, Jianxiu

Yasuda, Midori

Yasuhiro, SHINKAI

Ye, Jia-Hai

Ye, Jiandong

Ye, Min

Ye, Xingqian

Yeh, Jui-Ming

Yen, Gow-Chin

Yepuri, Nageshwar R.

Yerabolu, Jayasudhan Reddy

Yeung, Ying-yeung

Yi, Hankuil

Yi, Jianhua

Yi, Tae-hoo

Yi, Tao

Yi, Zhengping

Yiap, Beow Chin

Zhao, Yilei

Yim, Cheng-Bin

Yin, Hong

Yin, Mei-Chin

Yin, Ruohe

Yin, Shou-Wei
Yin, Shuang-Feng

Ying, Liu

Yokomatsu, Tsutomu

Yoneyama, Koichi

Yoo, Byoungseung

Yoo, Kyung Ho

Yoo, Yeong-Min

Yoo, Yung-Choon

Yoon, Do-Young

Yoon, Kyung Byung

Yoon, Moon-Young

Yoon, Seung Chul

Yordanov, Georgi

You, Liang

Yu, Yan-Ying

Yu, Guipeng

$\mathrm{Yu}, \mathrm{Li}$

$\mathrm{Yu}$, Longjiang

$\mathrm{Yu}$, Rongmin

Yu, Shi-shan

$\mathrm{Yu}$, Shuhui

Yu, Ting

Yu, Xiaoqi

Yu, Ying

Yu, Yinghua

Yu, Yongping

$\mathrm{Yu}$, Yuan-Hsiang

Yu, Yun-Qiu

Yu, Zhengkun

Yuan, Gu

Yue, Patrick Ying Kit

Yue, Yong-De

Yuliarti, Oni

Yun, Hyo-In

Yun, Junxian

Yun, Young Sook

Zabaras, Dimitrios

Zaferanloo, Bita

Zaikov, Gennadi

Zaleska, Adriana Zaleska

Zampieri, Daniele

Zapotoczna, Marta 
Zarejousheghani, Mashaalah

Zarrelli, Armando

Zavala, Jorge A.

Zavodnik, Ilya

Zaware, Nilesh

Zawilska, Jolanta B.

Zehl, Martin

Zeiner, Tim

Zelisko, Paul M.

Zemkova, H.

Zeng, Guang Ming

Zeng, $\mathrm{H}$.

Zeng, Runying

Zeng, Xiaoming

Zerbetto, Francesco

Zgoła-Grześkowiak, Agnieszka

Zhai, Haiyan

Zhang, Can

Zhang, Changhe

Zhang, Datong

Zhang, Dayi

Zhang, Dengsong

Zhang, De-Qiang

Zhang, Donglin

Zhang, Dong-ming

Zhang, Donna

Zhang, Guolin

Zhang, Guoqing

Zhang, Haifei

Zhang, Han-ting

Zhang, Hong-jie

Zhang, Jie

Zhang, Jinbiao

Zhang, Lin

Zhang, Min

Zhang, Ming-Rong

Zhang, Ning

Zhang, Qian

Zhang, Qingwen
Zhang, Shicheng

Zhang, Shijun

Zhang, Songping

Zhang, Tong

Zhang, X. Q.

Zhang, Xiang

Zhang, Xiaoming

Zhang, Xiaoying

Zhang, Xiaoyue

Zhang, Xiquan

Zhang, Xiuli

Zhang, Xiurong

Zhang, Yan

Zhang, Ying

Zhang, Yingrui

Zhang, Yong

Zhang, Yonghui

Zhang, Zhanhui

Zhang, Zhenwen

Zhao, Bao-xiang

Zhao, Bin

Zhao, Boxin

Zhao, Gui-sen

Zhao, Guohua

Zhao, Guoqiang

Zhao, Hong

Zhao, Hua

Zhao, Jian

Zhao, Jin-Hao

Zhao, Qinshi

Zhao, Wei

Zhao, Wei-Guang

Zhao, Weirong

Zhao, Xin-Qing

Zhao, Youxin

Zhao, Yuan

Zhao, Zhenjiang

Zheng, Jian-Bin

Zheng, Yu-Guo
Zhou, Zhongjun

Zhou, Guang-Biao

Zhou, Guang-Xiong

Zhou, Huchen

Zhou, Jia

Zhou, Tao

Zhou, Wei-Qing

Zhou, Xiaolong

Zhou, Yifa

Zhou, Zheng-Hong

Zhou, Zhi-Ming

Zhou, Zhongjun

Zhu, Changfu

Zhu, Fan

Zhu, Gangguo

Zhu, Guo-Yuan

Zhu, Jun

Zhu, Xiaofeng

Zhu, Xuan

Zhu, Ying

Zhu, Yinghuai

Zhu, Yulei

Zielske, Steven P.

Zitko, Jan

Złotek, Urszula

Zografos, Alandros L.

Zollo, Massimo

Zong, Xuxiao

Zorc, Branka

Zou, Zhongmei

Zoumpoulakis, Panagiotis

Zovko Končić, Marijana

Zrnčić, Mirta

Zughaier, Susu M.

Zuk, Magdalena

Zuo, Li

Zupkó, István

Zvyagi, Andrei V.

Zygadlo, Julio A.

(C) 2015 by the authors; licensee MDPI, Basel, Switzerland. This article is an open access article distributed under the terms and conditions of the Creative Commons Attribution license (http://creativecommons.org/licenses/by/4.0/). 